\title{
Holographic geometry for nonrelativistic systems emerging from generalized flow equations
}

\author{
Sinya Aoki, ${ }^{1, *}$ Shuichi Yokoyama, ${ }^{1, \dagger}$ and Kentaroh Yoshida ${ }^{2, \$}$ \\ ${ }^{1}$ Yukawa Institute for Theoretical Physics, Kyoto University, \\ Kitashirakawa Oiwake-cho, Sakyo-ku, Kyoto 606-8502, Japan \\ ${ }^{2}$ Department of Physics, Kyoto University, Kitashirakawa Oiwake-cho, Sakyo-kun, Kyoto 606-8502, Japan
}

(Received 21 February 2019; published 6 June 2019)

\begin{abstract}
An intriguing result presented by two of the present authors is that an anti-de Sitter space can be derived from a conformal field theory by considering a flow equation. A natural expectation is that given a certain data on the boundary system, the associated geometry would be able to emerge from a flow, even beyond the conformal case. As a step along this line, we examine this scenario for nonrelativistic systems with anisotropic scaling symmetries, such as Lifshitz field theories and Schrödinger invariant theories. In consequence we obtain a new hybrid geometry of Lifshitz and Schrödinger spacetimes as a general holographic geometry in this framework. We confirm that this geometry reduces to each of them by considering special nonrelativistic models.
\end{abstract}

DOI: 10.1103/PhysRevD.99.126002

\section{INTRODUCTION}

One of the fascinating subjects in string theory is a conjectured duality between a string (or gravity) theory on an anti-de Sitter (AdS) space in $d+1$ dimensions and a conformal field theory (CFT) in $d$ dimensions. This is called the AdS/CFT correspondence [1-3] and it is recognized as a realization of the holographic principle $[4,5]$. This correspondence has not been proven completely yet, but it is supported by a huge amount of circumstantial evidences and there is no contradiction so far.

The validity of the AdS/CFT correspondence has been well recognized nowadays, and it has opened up a new arena to consider applications of AdS/CFT to realistic systems in condensed matter physics (CMP) (often referred to as AdS/CMP). By following this approach, one can study the nonperturbative physics of gauge theories in a strongly coupled region by using a weakly coupled (semi-)classical gravity. Indeed, a lot of works have been carried out (For nice reviews, for example, see [6-11]).

One of the issues in the context of AdS/CMP is to consider how to realize nonrelativistic (NR) systems beyond the

\footnotetext{
saoki@yukawa.kyoto-u.ac.jp

† shuichi.yokoyama@yukawa.kyoto-u.ac.jp

"kyoshida@gauge.scphys.kyoto-u.ac.jp
}

Published by the American Physical Society under the terms of the Creative Commons Attribution 4.0 International license. Further distribution of this work must maintain attribution to the author(s) and the published article's title, journal citation, and DOI. Funded by SCOAP ${ }^{3}$. usual, relativistic CFT. A key ingredient is an anisotropic scaling like

$t \rightarrow \Lambda^{Z} t, \quad x^{i} \rightarrow \Lambda x^{i} \quad(i=1, \ldots, d-1)$,

$\Lambda$ : a real const.,

where $t$ and $x^{i}$ are time and spatial coordinates, respectively. Here $Z$ is called the dynamical critical exponent, which measures anisotropy of the system. In particular, the $Z=1$ case corresponds to the relativistic dilatation. There are two famous examples of symmetry algebra including the anisotropic scaling (1.1), Schrödinger algebra [12] and Lifshitz algebra. ${ }^{1}$ The geometries that preserve these symmetries as isometries have been constructed in [18-20], respectively. These geometries have been proposed from the symmetry argument. Then, with the standard dictionary (with some extension), the boundary theory is argued. One of the subtle points is that the boundary behavior of these geometries are not so well defined. Namely, a part of the geometry shrinks as we approach the boundary. Then, the supergravity approximation may not be valid any more and the notion of the boundary would be subtle.

Based on this observation, our motivation here is to consider the inverse direction to the preceding works. That is, we would like to demonstrate that a gravity dual may emerge starting from a nonrelativistic system with a scaling invariance. For this purpose, we shall follow the method based on flow equations proposed by two of the present

\footnotetext{
${ }^{1}$ For more details on the nonrelativistic algebras, see [13-17].
} 
TABLE I. Summary of the results in this paper. "LS," "R," "NR," "(C)PS” stand for "light-cone reversal symmetry," "relativistic," "nonrelativistic," "(conformal) primary scalar," respectively. $Z$ denotes the dynamical critical exponent.

\begin{tabular}{lllll}
\hline \hline Section & Theory type & Operator type & Flow equation type & Geometry type \\
\hline III & CFT & CPS & R & AdS \\
IV A & NRCFT & NRCPS & NR & NR Hybrid \\
IV B & CFT & CPS & NR & Lifshitz with $Z=2$ \\
IV C & NRCFT with LS & NRCPS & R & Schrödinger \\
IV D & CFT & NRCPS & R & Schrödinger \\
IV E & NRCFT & NRCPS & NR & Lifshitz with $Z=2$ \\
V & Lifshitz & Lifshitz PS & Lifshitz & Lifshitz with general $Z$ \\
\hline \hline
\end{tabular}

authors $[21,22] .^{2}$ In fact, they have derived an anti-de Sitter space from a CFT data by considering a flow equation. A natural expectation is that given a certain data on the boundary system, the associated geometry would be able to emerge from a flow, even beyond the conformal case. This possibility would open up an intriguing research arena to be studied in relation to the holographic principle.

In this paper, we examine this expectation for nonrelativistic systems with an anisotropic scaling symmetry, such as Lifshitz field theories and Schrödinger invariant theories. As a result we obtain a hybrid geometry of Schrödinger and Lifshitz spacetimes as a general holographic space by employing the associated two-point functions as the boundary data and generalizing the flow equation itself. This geometry contains both of them as special examples of nonrelativistic models. Our results are summarized in Table I.

The rest of this paper is organized as follows. Section II provides a brief review of gravity duals for nonrelativistic systems, Schrödinger spacetimes, and Lifshitz spacetimes. In Sec. III, we review how an AdS geometry emerges from a CFT data given at the boundary by employing a flow equation. In Secs. IV and V, we apply the formulation to nonrelativistic CFTs, a Lifshitz-type scale invariant theory, respectively. Section VI is devoted to conclusion and discussion. The Schrödinger algebra and the Lifshitz algebra are summarized in Appendix A, while a different flow equation is considered for the Lorentzian CFT in Appendix B. Transformation properties of the flowed field are given in Appendix C.

\section{GRAVITY DUALS FOR NONRELATIVISTIC SYSTEMS}

In this section, we shall give a brief review of gravity duals for nonrelativistic systems, Schrödinger spacetimes and Lifshitz spacetimes.

\section{A. Schrödinger spacetimes}

In 2008, Son proposed a geometry [18] preserving the Schrödinger symmetry as the maximal symmetry ${ }^{3}$ as a

\footnotetext{
${ }^{2}$ The flow equation method to construct a holographic theory was originally introduced in Ref. [23] and was further developed in Refs. [24,25].
}

holographic dual of a nonrelativistic system realized in a cold atom experiment. The metric is given by ${ }^{4}$

$\mathrm{d} s^{2}=\frac{ \pm 2 \mathrm{~d} x^{+} \mathrm{d} x^{-}+\sum_{i=1}^{d-1}\left(\mathrm{~d} x^{i}\right)^{2}+\mathrm{d} \tau^{2}}{\tau^{2}}-\sigma^{2} \frac{\left(\mathrm{d} x^{+}\right)^{2}}{\tau^{4}}$,

where $\sigma$ is a real constant parameter. This geometry can be considered as a one-parameter deformation of a $(d+2)$ dimensional AdS space, which is regarded as a gravity dual of a $d$-dimensional Schrödinger invariant system.

The Schrödinger algebra [12] is composed of a time translation, spatial translations, spatial rotations, Galilean boosts, a mass operator, a special conformal transformation and an anisotropic scaling as

$t \rightarrow \Lambda^{Z} t, \quad x^{i} \rightarrow \Lambda x^{i} \quad(i=1, \ldots, d-1)$,

$$
\Lambda \text { : a real const., }
$$

where $t$ and $x^{i}$ are time and spatial coordinates, respectively, and $Z$ is the dynamical critical exponent. In the original Schrödinger algebra, the $Z=2$ case is considered. In more general, one may consider an arbitrary value of $Z$, though the special conformal symmetry is broken except for $Z=2$ or 1 .

It is easy to see the invariance of the metric (2.1) under the Schrödinger symmetry (For the detail, see [18]). In particular, the metric (2.1) is invariant under the scaling

$$
\begin{aligned}
x^{+} & \rightarrow \Lambda^{2} x^{+}, \quad x^{-} \rightarrow x^{-}, \\
x^{i} & \rightarrow \Lambda x^{i} \quad(i=1, \ldots, d-1) . \quad \tau \rightarrow \Lambda \tau .
\end{aligned}
$$

\section{B. Lifshitz spacetimes}

The Lifshitz spacetime was proposed in [20] as a gravity dual for the Lifshitz fixed point realized in condensed matter systems. (For a comprehensive review of the Lifshitz

\footnotetext{
${ }^{3}$ The Schrödinger algebra can be embedded into a relativistic conformal algebra as a subalgebra. Hence the usual AdS metric is Schrödinger invariant, but this symmetry is not the maximal one. For the detail of the embedding, see Appendix A.

${ }^{4}$ The $\tau$ coordinate describes a radial direction, not the Euclidean time.
} 
holography, see [26].) The metric of the gravity dual is given by

$$
\mathrm{d} s^{2}=\frac{-\mathrm{d} t^{2}}{\tau^{2 Z}}+\frac{\sum_{i=1}^{d-1}\left(\mathrm{~d} x^{i}\right)^{2}+\mathrm{d} \tau^{2}}{\tau^{2}},
$$

which is invariant under the rescaling

$t \rightarrow \Lambda^{Z} t, \quad x^{i} \rightarrow \Lambda x^{i} \quad(i=1, \ldots, d-1), \quad \tau \rightarrow \Lambda \tau$.

This is a bulk realization of the scaling symmetry included in the Lifshitz algebra. As described in Appendix A in detail, the Lifshitz algebra is composed of a time translation, spatial translations, spatial rotations and the anisotropic scaling (2.2).

The metric (2.4) describes a $d+1$-dimensional bulk geometry and the boundary theory has $d-1$ spatial directions and a time direction.

\section{HOLOGRAPHIC GEOMETRY FROM A FLOWED CONFORMAL PRIMARY}

In Refs. [21,23], we proposed a mechanism for a holographic geometry to emerge from a boundary QFT coarse-grained by a flow equation. In this section, as a warm-up, we demonstrate this mechanism concretely by using conformal field theories.

\section{A. Euclidean case}

In this subsection, we review the emergence of an AdS geometry from a generic Euclidean CFT via the flow equation approach [21].

Consider a $D$-dimensional Euclidean CFT with a real scalar primary field $O_{0}(x)$ with conformal dimension $\Delta_{\mathcal{O}}$. By using the conformal symmetry of correlation functions, the 2-point function of the primary operator can be expressed as

$\left\langle O_{0}(x) O_{0}(0)\right\rangle=f_{0}\left(x^{2}\right), \quad f_{0}\left(\Lambda x^{2}\right)=\Lambda^{-\Delta_{\mathcal{O}}} f_{0}\left(x^{2}\right)$,

where $x^{2}:=\delta_{\mu \nu} x^{\mu} x^{\nu}$ and $f_{0}(u) \propto u^{-\Delta_{\mathcal{O}}}$.

Let us define a flowed field $\phi_{0}(x ; \eta)$ by a free flow equation as

$\frac{\partial \phi_{0}(x ; \eta)}{\partial \eta}=\partial^{2} \phi_{0}(x ; \eta), \quad \phi_{0}(x ; 0)=O_{0}(x), \quad \partial^{2}=\delta^{\mu \nu} \partial_{\mu} \partial_{\nu}$,

where $\eta$ is a positive number called a flow parameter, and the original field is recovered at the limit $\eta \rightarrow 0 .^{5}$ The formal solution of the flow equation is given by

\footnotetext{
${ }^{5}$ Note here that we naively take $\partial^{2}$ for the flow equation so as to realize a diffusion equation. This is the original choice employed in [21,23-25]. Only for this purpose, however, there may be some possibilities to generalize this choice, as we shall see later.
}

$$
\phi_{0}(x ; \eta)=e^{\eta \partial^{2}} O_{0}(x) .
$$

This is a well-defined operator, since this can be rewritten as

$$
\phi_{0}(x ; \eta)=\int d^{D} y K_{0}(x-y ; \eta) O_{0}(y)
$$

where

$$
K_{0}(x ; \eta):=\frac{1}{(4 \pi \eta)^{D / 2}} \exp \left[-\frac{x^{2}}{4 \eta}\right],
$$

is the Green function of the flow equation:

$$
\frac{\partial K_{0}(x ; \eta)}{\partial \eta}=\partial^{2} K_{0}(x ; \eta), \quad K_{0}(x ; 0)=\delta^{D}(x) .
$$

A virtue to coarse-grain operators by a flow equation is that the 2-point function of the flowed operator has no contact singularity. To see this, we compute the 2-point function of the flowed field $\phi_{0}$ as

$\left\langle\phi_{0}\left(x_{1} ; \eta_{1}\right) \phi_{0}\left(x_{2} ; \eta_{2}\right)\right\rangle=\mathrm{e}^{\eta_{1} \partial^{2}+\eta_{2} \partial^{\prime 2}}\left\langle O_{0}\left(x_{1}\right) O_{0}\left(x_{2}\right)\right\rangle$,

where we used (3.4) and $\partial, \partial^{\prime}$ act on $x_{1}, x_{2}$, respectively: $\partial_{\mu}:=\frac{\partial}{\partial x_{1}^{\mu}}, \partial_{\mu}^{\prime}:=\frac{\partial}{\partial x_{2}^{\mu}}$. By using (3.1), we can rewrite this as

$$
\left\langle\phi_{0}\left(x_{1} ; \eta_{1}\right) \phi_{0}\left(x_{2} ; \eta_{2}\right)\right\rangle=\mathrm{e}^{\eta_{+} \partial^{2}} f_{0}\left(x_{12}^{2}\right),
$$

where $\eta_{+}:=\eta_{1}+\eta_{2}, x_{12}:=x_{1}-x_{2}$. This quantity is a smooth function of $x_{12}^{2}$ and $\eta_{+}$since this can be rewritten by using the Green function (3.5), which implies the absence of the contact singularity, as was claimed. ${ }^{6}$ Let us denote this smooth function by $F_{0}\left(x_{12}^{2} ; \eta_{+}\right)$. Then by using the scaling relation in (3.1) the function $F_{0}$ satisfies

$$
F_{0}\left(\Lambda^{2} x_{12}^{2} ; \Lambda^{2} \eta_{+}\right)=\Lambda^{-2 \Delta_{\mathcal{O}}} F_{0}\left(x_{12}^{2} ; \eta_{+}\right) .
$$

Choosing $\Lambda=1 / \eta_{+}^{\frac{1}{2}}$ we find

$$
\left\langle\phi_{0}\left(x_{1} ; \eta_{1}\right) \phi_{0}\left(x_{2} ; \eta_{2}\right)\right\rangle=\frac{1}{\eta_{+}^{\Delta_{\mathcal{O}}}} F_{0}\left(\frac{x_{12}^{2}}{\eta_{+}} ; 1\right) .
$$

We introduce a normalized field denoted by $\sigma_{0}$ as

$$
\sigma_{0}(x ; \eta):=\frac{\phi_{0}(x ; \eta)}{\sqrt{\left\langle\phi_{0}(x ; \eta)^{2}\right\rangle}},
$$

so that $\left\langle\sigma_{0}(x ; \eta)^{2}\right\rangle=1$. The average is taken in the original $D$ dimensional theory. This is well defined due to the fact that the contact singularity is resolved. Using the normalized field we define

\footnotetext{
${ }^{6}$ The explicit form was given in [21].
} 


$$
\begin{gathered}
\hat{g}_{A B}(x ; \eta) \equiv R^{2} \lim _{\left(x^{\prime}, \eta^{\prime}\right) \rightarrow(x, \eta)} \frac{\partial}{\partial z^{A}} \sigma_{0}(x ; \eta) \frac{\partial}{\partial z^{\prime B}} \sigma_{0}\left(x^{\prime} ; \eta^{\prime}\right), \\
g_{A B}(z) \equiv\left\langle\hat{g}_{A B}(x ; \eta)\right\rangle,
\end{gathered}
$$

where $R$ is a certain length scale, $\left(z^{A}\right)=\left(x^{\mu}, \tau\right)$ with $\tau:=$ $\sqrt{\eta / \alpha}$ and $\alpha$ is fixed later. Thanks to this normalization, $g_{A B}(z)$ becomes the information metric [21], and thus can be regarded as a metric in the $D+1$ dimensional holographic space, called the induced metric. For simplicity, we take $R=1$ hereafter.

The 2-point function of the normalized filed $\sigma_{0}$ becomes

$$
\left\langle\sigma_{0}\left(x_{1} ; \eta_{1}\right) \sigma_{0}\left(x_{2} ; \eta_{2}\right)\right\rangle=\left(\frac{2 \sqrt{\eta_{1} \eta_{2}}}{\eta_{+}}\right)^{\Delta_{\mathcal{O}}} G_{0}\left(\frac{x_{12}^{2}}{\eta_{+}}\right),
$$

where $G_{0}(u):=F_{0}(u ; 1) / F_{0}(0 ; 1)$, and thus $G_{0}(0)=1$. Therefore the induced metric is evaluated as

$$
g_{\mu \nu}(z)=-\delta_{\mu \nu} \frac{1}{\alpha \tau^{2}} G_{0}^{\prime}(0), \quad g_{\tau \tau}(z)=\frac{\Delta_{\mathcal{O}}}{\tau^{2}},
$$

and the other components vanish.

We can determine $G^{\prime}(0)$ by using the flow equation for the 2-point function of the normalized operator

$\partial_{\eta_{1}}\left\langle\sigma_{0}\left(x_{1} ; \eta_{1}\right) \sigma_{0}\left(x_{2} ; \eta_{2}\right)\right\rangle=\partial_{x_{1}}^{2}\left\langle\sigma_{0}\left(x_{1} ; \eta_{1}\right) \sigma_{0}\left(x_{2} ; \eta_{2}\right)\right\rangle$,

which leads to

$$
\begin{aligned}
- & \frac{\Delta_{\mathcal{O}}}{\eta_{+}^{\Delta_{\mathcal{O}}+1}} G_{0}\left(\frac{x_{12}^{2}}{\eta_{+}}\right)-\frac{x_{12}^{2}}{\eta_{+}^{\Delta_{\mathcal{O}}+2}} G_{0}^{\prime}\left(\frac{x_{12}^{2}}{\eta_{+}}\right) \\
& =\frac{2 D}{\eta_{+}^{\Delta_{\mathcal{O}}+1}} G_{0}^{\prime}\left(\frac{x_{12}^{2}}{\eta_{+}}\right)+\frac{4 x_{12}^{2}}{\eta_{+}^{\Delta_{\mathcal{O}}+2}} G_{0}^{\prime \prime}\left(\frac{x_{12}^{2}}{\eta_{+}}\right) .
\end{aligned}
$$

From this we find

$$
G_{0}^{\prime}(0)=-\frac{\Delta_{\mathcal{O}}}{2 D}
$$

Therefore, taking $\alpha=1 /(2 D)$, we obtain the Euclidean AdS metric as

$$
\mathrm{d} s^{2}=\Delta_{\mathcal{O}} \frac{\mathrm{d} x^{2}+\mathrm{d} \tau^{2}}{\tau^{2}}
$$

Thus, the flow approach generates an AdS space from a Euclidean CFT.

This result can be confirmed from symmetry argument. To see this let us consider the $D$ dimensional conformal transformation of the normalized field:

$$
\begin{aligned}
\delta^{\text {conf }} \sigma_{0}(x ; \eta)= & -\left\{\eta\left(\partial^{2} \delta x^{\mu}\right)+2 \eta^{2}\left(\partial^{\nu} \partial^{\rho} \delta x^{\mu}\right) \partial_{\nu} \partial_{\rho}\right. \\
+ & \left.2 \eta\left(\partial^{\nu} \delta x^{\mu}\right) \partial_{\nu}+\delta x^{\mu}\right\} \partial_{\mu} \sigma_{0}(x ; \eta) \\
& -\frac{\Delta_{\mathcal{O}}}{D}\left\{2 \eta\left(\partial^{\nu} \partial_{\mu} \delta x^{\mu}\right) \partial_{\nu}+\left(\partial_{\mu} \delta x^{\mu}\right)\right\} \sigma_{0}(x ; \eta) .
\end{aligned}
$$

Following [21], we decompose this into isometries of the $D+1$ dimensional AdS and the rest as

$$
\delta^{\text {conf }} \sigma_{0}(x ; \eta)=\delta^{\text {diff }} \sigma_{0}(x ; \eta)+\delta^{\text {extra }} \sigma_{0}(x ; \eta),
$$

where

$$
\begin{aligned}
\delta^{\text {diff }} \sigma_{0}(x ; \eta) & =-\left(\bar{\delta} \eta \partial_{\eta}+\bar{\delta} x^{\mu} \partial_{\mu}\right) \sigma_{0}(x ; \eta), \\
\delta^{\text {extra }} \sigma_{0}(x ; \eta) & =4 \eta^{2} b^{\nu} \partial_{\nu}\left(\partial_{\eta}+\frac{\Delta_{\mathcal{O}}+2}{2 \eta}\right) \sigma_{0}(x ; \eta),
\end{aligned}
$$

with

$\bar{\delta} x^{\mu}=\delta x^{\mu}+2 D \eta b^{\mu}, \quad \bar{\delta} \eta=\left(2 \lambda-4\left(b_{\mu} x^{\mu}\right)\right) \eta$.

Then the conformal transformation of the induced metric is computed as

$$
\begin{aligned}
\delta^{\text {conf }} g_{A B}(z)= & \delta^{\text {diff }} g_{A B}(z) \\
& +\lim _{\left(x^{\prime} ; \eta^{\prime}\right) \rightarrow(x ; \eta)} \frac{\partial}{\partial z^{A}} \frac{\partial}{\partial z^{\prime B}}\left\langle\delta^{\text {extra }} \sigma_{0}(x ; \eta) \sigma_{0}\left(x^{\prime} ; \eta^{\prime}\right)\right. \\
& \left.+\sigma_{0}(x ; \eta) \delta^{\text {extra }} \sigma_{0}\left(x^{\prime} ; \eta^{\prime}\right)\right\rangle,
\end{aligned}
$$

where

$$
\begin{aligned}
\delta^{\text {conf }} g_{A B}(z) & :=\left\langle\delta^{\text {conf }} \hat{g}_{A B}(x ; \eta)\right\rangle, \\
\delta^{\text {diff }} g_{A B}(z) & :=\left\langle\delta^{\text {diff }} \hat{g}_{A B}(x ; \eta)\right\rangle .
\end{aligned}
$$

Since the 2-point correlation function is invariant under an arbitrary conformal transformation, $\delta^{\text {conf }} g_{A B}(z)=0$. On the other hand, by using (3.14) and (3.22) we find

$$
\begin{aligned}
& \left\langle\delta^{\text {extra }} \sigma_{0}\left(x_{1} ; \eta_{1}\right) \sigma_{0}\left(x_{2} ; \eta_{2}\right)+\sigma_{0}(x ; \eta) \delta^{\text {extra }} \sigma_{0}\left(x_{2} ; \eta_{2}\right)\right\rangle \\
& =-8 \frac{\left(\sqrt{4 \eta_{1} \eta_{2}}\right)^{\Delta_{\mathcal{O}}}}{\eta_{+}^{\Delta_{\mathcal{O}}+2}}\left(\eta_{1}-\eta_{2}\right)\left(b \cdot x_{12}\right) x_{12}^{2} G_{0}^{\prime \prime}\left(\frac{x_{12}^{2}}{\eta_{+}}\right),
\end{aligned}
$$

where we set $\alpha \cdot \beta:=\alpha^{\mu} \beta_{\mu}$. This implies that the 2 nd term in (3.24) vanishes. Therefore

$$
\delta^{\text {diff }} g_{A B}(z)=0 .
$$

Since this result means that the metric is invariant under the isometry of $\operatorname{AdS}\left(\delta^{\mathrm{diff}}, \bar{\delta}\right)$, which is a maximally symmetric 
space, the induced metric has to be an AdS one up to an overall constant. This is the desired result.

\section{B. Lorentzian case}

In this subsection we comment on the construction of a Lorentzian AdS geometry by a flow equation approach. To this end the first thing to do is to smear operators by a flow equation in the Lorentzian space. This should be done so as to preserve the causal structure at least in a certain amount. Although it is unclear whether such a smearing is possible by a diffusion-type differential equation, a natural candidate of a Lorentzian free flow equation may be given by

$$
\frac{\partial \phi_{0}(x ; \eta)}{\partial \eta}=\partial^{2} \phi_{0}(x ; \eta), \quad \partial^{2}=g^{\mu \nu} \partial_{\mu} \partial_{\nu}
$$

with $g^{\mu \nu}=\operatorname{diag}(-1,1, \ldots, 1)$. However it is soon realized that the formal solution of this flow equation becomes divergent.

To avoid this problem, in what follows, we simply use the Wick rotation for a time coordinate. Then the Lorentzian flow equation (3.28) is mapped in the Euclidean one (3.2), which can be solved without any problems. ${ }^{7}$ Then we rotate the result back to the Lorentzian space. ${ }^{8}$

Let us start the flow equation in the Euclidean space (3.2). For later purposes we introduce a complex coordinate that $x=\left(\vec{x}, x^{+}, x^{-}\right)$with $x^{ \pm}=\left(x^{D-1} \pm i x^{D}\right) / \sqrt{2}$ and rewrite the flow equation as

$\frac{\partial \phi_{0}(x ; \eta)}{\partial \eta}=\partial^{2} \phi_{0}(x ; \eta), \quad \partial^{2}=\vec{\partial}^{2}+2 \partial_{+} \partial_{-}$.

The flowed field is expressed as

$$
\begin{aligned}
& \phi_{0}(x ; \eta)=\int d^{D} y K_{0}(x-y ; \eta) O_{0}(y) \\
& K_{0}(x ; \eta)=\frac{1}{(4 \pi \eta)^{D / 2}} \exp \left[-\frac{\vec{x}^{2}+2 x^{+} x^{-}}{4 \eta}\right] .
\end{aligned}
$$

The 2-point function of the normalized field is given by

$$
\begin{aligned}
& \left\langle\sigma_{0}\left(t_{1}, \vec{x}_{1} ; \eta_{1}\right) \sigma_{0}\left(t_{2}, \vec{x}_{2} ; \eta_{2}\right)\right\rangle \\
& \quad=\left(\frac{2 \sqrt{\eta_{1} \eta_{2}}}{\eta_{+}}\right)^{\Delta_{\mathcal{O}}} G_{0}\left(\frac{\vec{x}^{2}+2 x^{+} x^{-}}{\eta_{+}}\right),
\end{aligned}
$$

which leads to

\footnotetext{
${ }^{7}$ The AdS/CFT correspondence in the Lorentzian space has a different aspect from the Euclidean case [27-29]. For example, see [30] for a careful study on the analytic continuation in this context.

${ }^{8}$ This approach has a virtue that Lorentz invariance is manifest, but assumes a sufficient fall-off of a flowed operator at infinity, which is nontrivial for any operators in CFT. We instead present a different approach to this problem in Appendix B.
}

$g_{i j}=-\delta_{i j} \frac{G_{0}^{\prime}(0)}{\alpha \tau^{2}}, \quad g_{+-}=g_{-+}=-\frac{G_{0}^{\prime}(0)}{\alpha \tau^{2}}, \quad g_{\tau \tau}=\frac{\Delta_{\mathcal{O}}}{\tau^{2}}$,

and the others vanish. By using (3.18) and $\alpha=1 /(2 D)$, this gives the Euclidean AdS metric containing a complex coordinate

$$
\mathrm{d} s^{2}=\Delta_{\mathcal{O}} \frac{\mathrm{d} \vec{x}^{2}+2 \mathrm{~d} x^{+} \mathrm{d} x^{-}+\mathrm{d} \tau^{2}}{\tau^{2}}
$$

By the analytic continuation $x^{D}=-i x^{0}$, the coordinates $x^{+}, x^{-}$become the light-cone ones $x^{ \pm}=\left(x^{D-1} \pm x^{0}\right) / \sqrt{2}$, which converts the Euclidean AdS metric to the Lorentzian one with the formally same expression.

\section{HOLOGRAPHIC GEOMETRY FROM FLOWED NONRELATIVISTIC CONFORMAL PRIMARIES}

In this section we shall apply the flow equation approach (presented in Sec. III) to a nonrelativistic conformal primary operator, and investigate induced geometry by a nonrelativistic flow equation.

\section{A. General induced geometry}

In this subsection we investigate a general holographic geometry for a generic nonrelativistic CFT with a nonrelativistic flow equation. To this end we start with a $d$ dimensional nonrelativistic CFT with a primary scalar field $O(\vec{x}, t)$ with a general dimension $\Delta_{\mathcal{O}}$. The nonrelativistic conformal symmetry constrains the 2-point function of this operator as

$$
\left\langle O\left(\vec{x}_{1}, t_{1}\right) O^{\dagger}\left(\vec{x}_{2}, t_{2}\right)\right\rangle=\frac{1}{t_{12}^{\Delta_{\mathcal{O}}}} f\left(\frac{\vec{x}_{12}^{2}}{2 t_{12}}\right),
$$

where $\vec{x}^{2}=x_{i} x^{i}$.

For our argument, let us introduce an extra direction denoted by $x^{-}$, in order to embed the $d$-dimensional nonrelativistic symmetry into the $D=d+1$ dimensional relativistic conformal symmetry, generated with

$$
\delta x^{\mu}=a^{\mu}+\omega^{\mu}{ }_{\nu} x^{\nu}+\lambda x^{\mu}+b^{\mu} x^{2}-2 x^{\mu}(b \cdot x),
$$

where $a^{\mu}, \omega^{\mu \nu}, \lambda$ and $b^{\mu}$ are parameters of translation, rotation, dilatation and special conformal transformation, respectively, and the $D$ dimensional light-cone coordinate is given by $x:=\left(x^{\mu}\right)=\left(\vec{x}, x^{+}, x^{-}\right)$with $x^{+}=t$. The transformation law under the Schrödinger symmetry can be derived from the conformal transformation (4.2) for a scalar primary operator with dimension $\Delta_{\mathcal{O}}$,

$\delta^{\mathrm{conf}} O_{0}(x)=-\delta x^{\mu} \partial_{\mu} O_{0}(x)-\frac{\Delta_{\mathcal{O}}}{D}\left(\partial_{\mu} \delta x^{\mu}\right) O_{0}(x)$, 
by keeping only the following components of the parameters,

$a^{i}, \quad a^{+}, \quad a^{-} \equiv \mu, \quad \omega_{j}^{i}, \quad \omega^{-i} \equiv v^{i}, \quad \omega^{+-} \equiv \lambda, \quad b^{-} \equiv b$,

and the other parameters are set to zero. Then the transformation law (4.2) reduces to

$$
\begin{aligned}
\delta^{s} x^{i} & =a^{i}+\omega_{j}^{i} x^{j}-v^{i} x^{+}+\lambda x^{i}-2 b x^{i} x^{+}, \\
\delta^{s} x^{+} & =a^{+}+2 \lambda x^{+}-2 b\left(x^{+}\right)^{2}, \\
\delta^{s} x^{-} & =\mu+v^{i} x^{i}+b \vec{x}^{2} .
\end{aligned}
$$

Here $v^{i}, \mu, \lambda$ and $b$ correspond to parameters of the Galilei transformation, mass operator, scale transformation and special conformal transformation, respectively. Note here that the $x^{-}$coordinate does not appear on the right-hand sides. This means that the translation for the $x^{-}$direction commutes with the other transformations, so that the associated generator $i \partial_{-}$is identified with the mass operator. Notably, $x^{+}$corresponds to the time direction in the nonrelativistic system.

Thus a scalar primary operator $O(x)$ with dimension $\Delta_{\mathcal{O}}$ transforms as

$$
\begin{aligned}
\delta^{S} O(x)= & {\left[-a^{i} \partial_{i}-a^{+} \partial_{+}-\mu \partial_{-}-\omega^{i j} x^{j} \partial_{i}\right.} \\
& -v^{i}\left(x_{i} \partial_{-}-x^{+} \partial_{i}\right)-\lambda\left(\Delta_{O}+x^{i} \partial_{i}+2 x^{+} \partial_{+}\right) \\
& \left.+b\left\{-\left(x^{i}\right)^{2} \partial_{-}+2 x^{+}\left(\Delta_{\mathcal{O}}+x^{i} \partial_{i}+x^{+} \partial_{+}\right)\right\}\right] O(x),
\end{aligned}
$$

and its 2-point function is given by

$$
\left\langle O\left(\vec{x}_{1}, x_{1}^{+}, x_{1}^{-}\right) O^{\dagger}\left(\vec{x}_{2}, x_{2}^{+}, x_{2}^{-}\right)\right\rangle=\frac{1}{\left(x_{12}^{+}\right)^{\Delta_{\mathcal{O}}}} f\left(x_{12}^{-}+\frac{\vec{x}_{12}^{2}}{2 x_{12}^{+}}\right) .
$$

It is easy to see that (4.7) reduces to (4.1) when $x_{12}^{-} \rightarrow 0$.

We smear this nonrelativistic conformal primary operator by a nonrelativistic flow equation. A general nonrelativistic free flow equation is

$$
\begin{aligned}
\frac{\partial}{\partial \eta} \phi(x ; \eta) & =\left(2 i \bar{m} \partial_{+}+2 \partial_{-} \partial_{+}+\vec{\partial}^{2}\right) \phi(x ; \eta), \\
\phi(x ; 0) & =O(x),
\end{aligned}
$$

where $\bar{m}$ is a real parameter of mass dimension one. This can be solved as

$$
\begin{aligned}
\phi(x ; \eta) & =e^{\eta\left(2 i \bar{m} \partial_{+}+2 \partial_{-} \partial_{+}+\vec{\partial}^{2}\right)} O(x) \\
& =\int d^{D} x^{\prime} K\left(x-x^{\prime} ; \eta\right) O\left(x^{\prime}\right)
\end{aligned}
$$

where

$$
K(x ; \eta)=\exp \left[-i \bar{m} x^{-}\right] \frac{e^{\frac{-2 x^{+} x^{-}-\bar{x}^{2}}{4 \eta}}}{\sqrt{4 \pi \eta} D} .
$$

Let us study the transformation of the flowed operator under the nonrelativistic conformal transformation.

$$
\begin{aligned}
\delta_{\bar{m}}^{S} \phi(x ; \eta) & =e^{\eta\left(2 i \bar{m} \partial_{+}+2 \partial_{-} \partial_{+}+\vec{\partial}^{2}\right)} \delta^{S} O(x) \\
& =\delta^{S} \phi(x ; \eta)+{\delta_{\bar{m}}}^{S} \phi(x ; \eta)
\end{aligned}
$$

where $\delta^{S}$ is given by (4.6) and

$$
\begin{aligned}
\delta_{\bar{m}}^{\prime S} \phi(x ; \eta)= & {\left[2 i \bar{m} \eta v^{i} \partial_{i}-\lambda 2 \eta \partial_{\eta}\right.} \\
& +b\left\{\left(-2 \eta(d-1)+4 \eta \Delta_{\mathcal{O}}+4 \eta^{2} \partial_{\eta}\right) \partial_{-}\right. \\
& +4 i \bar{m} \eta\left(\Delta_{\mathcal{O}}+x^{i} \partial_{i}+\eta \vec{\partial}^{2}+\eta \partial_{\eta}\right) \\
& \left.\left.+4 x^{+} \eta \partial_{\eta}\right\}\right] \phi(x ; \eta) .
\end{aligned}
$$

Note that the flowed operator $\phi(x ; \eta)$ transforms differently from $O(x)$ under the Galilei transformation unless $\bar{m}$ vanishes.

We move on to the 2-point function of the flowed operator, which is written as

$$
\begin{aligned}
& \left\langle\phi\left(x_{1} ; \eta_{1}\right) \phi^{\dagger}\left(x_{2} ; \eta_{2}\right)\right\rangle \\
& =e^{\eta_{1}\left(2 i \bar{m} \partial_{+}+2 \partial_{-} \partial_{+}+\vec{\partial}^{2}\right)+\eta_{2}\left(-2 i \bar{m} \partial_{+}^{\prime}+2 \partial_{-}^{\prime} \partial_{+}^{\prime}+\vec{\partial}^{\prime 2}\right)}\left\langle O\left(x_{1}\right) O^{\dagger}\left(x_{2}\right)\right\rangle \\
& =\exp \left[\eta_{+}\left(2 i \bar{m} \partial_{+}+2 \partial_{-} \partial_{+}+\vec{\partial}^{2}\right)\right]\left[\frac{f\left(x_{12}^{-}+\frac{\vec{x}_{12}^{2}}{2 x_{12}^{+}}\right)}{\left(x_{12}^{+}\right)^{\Delta_{\mathcal{O}}}}\right]
\end{aligned}
$$

where we used the same notation in the previous section. This function can be written as $F_{1}\left(\left(\vec{x}_{12}\right)^{2}, x_{12}^{+}, x_{12}^{-} ; \eta_{+}\right)$. From the Galilean invariance we find

$v^{i}\left(x^{i} \partial_{-}-\left(x^{+}+2 i \bar{m} \eta\right) 2 x^{i} \partial_{\vec{x}^{2}}\right) F_{1}\left(\vec{x}^{2}, x^{+}, x^{-} ; \eta\right)=0$.

This can be generally solved by

$F_{1}\left(\vec{x}^{2}, x^{+}, x^{-} ; \eta\right)=F\left(2\left(x^{+}+2 i \bar{m} \eta\right) x^{-}+\vec{x}^{2}, x^{+} ; \eta\right)$,

where $F$ is an unknown function. The nonrelativistic scale invariance requires

$F_{1}\left(\lambda^{2} \vec{x}^{2}, \lambda^{2} x^{+}, x^{-} ; \lambda^{2} \eta_{+}\right)=\lambda^{-2 \Delta_{\mathcal{O}}} F_{1}\left(\vec{x}^{2}, x^{+}, x^{-} ; \eta_{+}\right)$,

which constrains the function in such a way that

$$
\begin{aligned}
F & \left(2\left(x^{+}+2 i \bar{m} \eta_{+}\right) x^{-}+\vec{x}^{2}, x^{+} ; \eta_{+}\right) \\
& =\frac{1}{\eta_{+}^{\Delta_{\mathcal{O}}}} F\left(\frac{2\left(x^{+}+2 i \bar{m} \eta_{+}\right) x^{-}+\vec{x}^{2}}{\eta_{+}}, \frac{x^{+}}{\eta_{+}} ; 1\right) .
\end{aligned}
$$


As a result the 2-point function is written as

$$
\begin{aligned}
& \left\langle\phi\left(x_{1} ; \eta_{1}\right) \phi^{\dagger}\left(x_{2} ; \eta_{2}\right)\right\rangle \\
& =\frac{1}{\eta_{+}^{\Delta_{\mathcal{O}}}} F\left(\frac{2\left(x_{12}^{+}+2 i \bar{m} \eta_{+}\right) x_{12}^{-}+\left(\vec{x}_{12}\right)^{2}}{\eta_{+}}, \frac{x_{12}^{+}}{\eta_{+}} ; 1\right) .
\end{aligned}
$$

Note that the contact singularity is resolved with a general parametrization.

By using this result the 2-point function of the normalized field becomes

$$
\begin{aligned}
& \left\langle\sigma\left(x_{1} ; \eta_{1}\right) \sigma^{\dagger}\left(x_{2} ; \eta_{2}\right)\right\rangle \\
& =\left(\frac{4 \eta_{1} \eta_{2}}{\eta_{+}^{2}}\right)^{\Delta_{\mathcal{O}} / 2} G\left(\frac{2\left(x_{12}^{+}+2 i \bar{m} \eta_{+}\right) x_{12}^{-}+\left(\vec{x}_{12}\right)^{2}}{\eta_{+}}, \frac{x_{12}^{+}}{\eta_{+}}\right),
\end{aligned}
$$

where $G(u, v)=F(u, v ; 1) / F(0,0 ; 1)$.

Since the normalized flowed field $\sigma$ is complex-valued for nonrelativistic theories in general, the definition of the metric operator, for example, should be modified like

$\hat{g}_{A B}(x ; \eta)=\frac{\partial_{A} \sigma(x ; \eta) \partial_{B} \sigma^{\dagger}(x ; \eta)+\partial_{B} \sigma(x ; \eta) \partial_{A} \sigma^{\dagger}(x ; \eta)}{2}$,

so that the induced metric, which is given by $g_{A B}(z)=$ $\left\langle\hat{g}_{A B}(x ; \eta)\right\rangle$, becomes real and symmetric. ${ }^{9}$ Then the induced metric is computed as

$$
\begin{gathered}
g_{\eta \eta}(z)=\frac{\Delta_{\mathcal{O}}}{4 \eta^{2}}, \quad g_{+\eta}(z)=g_{-\eta}(z)=0, \\
g_{++}(z)=\frac{-1}{4 \eta^{2}} G^{(0,2)}(\overrightarrow{0}), \\
g_{+-}(z)=\frac{-G^{(1,0)}(\overrightarrow{0})-2 i \bar{m} G^{(1,1)}(\overrightarrow{0})}{\eta}, \\
g_{--}(z)=-(4 i \bar{m})^{2} G^{(2,0)}(\overrightarrow{0}), \quad g_{i j}(z)=\frac{-\delta_{i j}}{\eta} G^{(1,0)}(\overrightarrow{0}),
\end{gathered}
$$

where $G^{(n, m)}(u, v):=\partial_{u}^{n} \partial_{v}^{m} G(u, v)$. The undetermined constants are not independent from each other, since the flow equation implies

\footnotetext{
${ }^{9}$ If one wants to relate the induced metric to an information metric for a complex-valued vector model as in [21], an extra term $\frac{1}{2}\left(\left\langle\sigma^{\dagger} \partial_{A} \sigma\right\rangle\left\langle\sigma^{\dagger} \partial_{B} \sigma\right\rangle+\left\langle\partial_{A} \sigma^{\dagger} \sigma\right\rangle\left\langle\partial_{B} \sigma^{\dagger} \sigma\right\rangle\right)$ is necessary to add to the definition of the induced metric. However, this term may not be written as an expectation value of a specific operator. Hence we shall avoid using this definition here, probably though the relevance to an information metric would be significant for the bulk description as shown in [21]. We shall leave this issue as a future work. We appreciate Janos Balog for discussion on this point.
}

$$
\begin{aligned}
& \partial_{\eta}\left\langle\phi(x ; \eta) \phi^{\dagger}\left(x^{\prime} ; \eta^{\prime}\right)\right\rangle \\
& \quad=\left(2\left(i \bar{m}+\partial_{-}\right) \partial_{+}+\partial_{i}^{2}\right)\left\langle\phi(x ; \eta) \phi^{\dagger}\left(x^{\prime} ; \eta^{\prime}\right)\right\rangle .
\end{aligned}
$$

From this we obtain

$$
-\Delta_{\mathcal{O}}=(2 d+2) G^{(1,0)}(\overrightarrow{0})+8 i \bar{m} G^{(1,1)}(\overrightarrow{0})+2 i \bar{m} G^{(0,1)}(\overrightarrow{0})
$$

$$
\begin{aligned}
\left(-\Delta_{\mathcal{O}}-1\right) G^{(1,0)}(\overrightarrow{0})= & (2 d+6) G^{(2,0)}(\overrightarrow{0})+8 i \bar{m} G^{(2,1)}(\overrightarrow{0}) \\
& +2 i \bar{m} G^{(1,1)}(\overrightarrow{0}) .
\end{aligned}
$$

The induced line element is thus written as

$$
\begin{aligned}
d s^{2}= & \frac{\Delta_{\mathcal{O}}}{4 \eta^{2}} d \eta^{2}+\frac{-G^{(0,2)}(\overrightarrow{0})}{4 \eta^{2}}\left(d x^{+}\right)^{2} \\
& +2 \frac{-G^{(1,0)}(\overrightarrow{0})-2 i \bar{m} G^{(1,1)}(\overrightarrow{0})}{\eta} d x^{+} d x^{-} \\
& +(4 \bar{m})^{2} G^{(2,0)}(\overrightarrow{0})\left(d x^{-}\right)^{2}+\frac{-\delta_{i j} G^{(1,0)}(\overrightarrow{0})}{\eta} d x^{i} d x^{j}
\end{aligned}
$$

We refer to this geometry as the nonrelativistic (NR) hybrid geometry. In what follows, we apply this result to specific examples.

\section{B. Nonrelativistic flow of a conformal primary}

A first application is to nonrelativistic smearing of a conformal primary scalar operator in a general CFT considered in Sec. III. In this case the induced geometry is more simplified than (4.26).

To see this let us smear the conformal primary scalar field $O_{0}(x)$ by the nonrelativistic flow equation (4.8) and denote the smeared operator by $\phi_{\bar{m}}(x ; \eta)$. The relation between the relativistic flowed operator and the nonrelativistic one is

$$
\phi_{\bar{m}}(x ; \eta)=e^{2 i \eta \bar{m} \partial_{+}} \phi_{0}(x ; \eta)
$$

Therefore the 2-point function of the flowed operator is

$$
\begin{aligned}
& \left\langle\phi_{\bar{m}}\left(x_{1} ; \eta_{1}\right) \phi_{\bar{m}}^{\dagger}\left(x_{2} ; \eta_{2}\right)\right\rangle \\
& \quad=e^{2 i \eta_{1} \bar{m} \partial_{+}} e^{-2 i \eta_{2} \bar{m} \partial_{+}^{+}}\left\langle\phi_{0}\left(x_{1} ; \eta_{1}\right) \phi_{0}\left(x_{2} ; \eta_{2}\right)\right\rangle \\
& \quad=\frac{1}{\eta_{+}^{\Delta_{\mathcal{O}}}} F_{0}\left(\frac{2\left(x_{12}^{+}+2 i \eta_{+} \bar{m}\right) x_{12}^{-}+\left(\vec{x}_{12}\right)^{2}}{\eta_{+}} ; 1\right),
\end{aligned}
$$

and that of the normalized field is 


$$
\begin{aligned}
& \left\langle\sigma_{\bar{m}}\left(x_{1} ; \eta_{1}\right) \sigma_{\bar{m}}^{\dagger}\left(x_{2} ; \eta_{2}\right)\right\rangle \\
& \quad=\left(\frac{2 \sqrt{\eta_{1} \eta_{2}}}{\eta_{+}}\right)^{\Delta_{\mathcal{O}}} G_{0}\left(\frac{2\left(x_{12}^{+}+2 i \eta_{+} \bar{m}\right) x_{12}^{-}+\left(\vec{x}_{12}\right)^{2}}{\eta_{+}}\right) .
\end{aligned}
$$

$G(u, v)$ in Sec. IV A now reduces to $G_{0}(u)$ so that

$$
G^{(n, 0)}(\overrightarrow{0})=G_{0}^{(n)}(0), \quad G^{(n, k)}(\overrightarrow{0})=0,
$$

for $n \in \mathbb{Z}_{\geq 0}, k \in \mathbb{Z}_{\geq 1}$. Then (4.25) reduces to

$$
G^{(2,0)}(\overrightarrow{0})=-\frac{\Delta_{\mathcal{O}}+1}{2(D+2)} G^{\prime}(0)=\frac{\Delta_{\mathcal{O}}\left(\Delta_{\mathcal{O}}+1\right)}{4 D(D+2)}
$$

where $D=d+1$. As a result the induced line element given by (4.26) reduces to

$$
d s^{2}=\Delta_{\mathcal{O}}\left[\gamma\left(d x^{-}\right)^{2}+\frac{d \tau^{2}+2 d x^{+} d x^{-}+d \vec{x}^{2}}{\tau^{2}}\right]
$$

where we set

$$
\tau \equiv \sqrt{2 D \eta}, \quad \gamma \equiv 4 \bar{m}^{2} \frac{\Delta_{\mathcal{O}}+1}{D(D+2)}
$$

both of which are positive. Note that the limit $\bar{m} \rightarrow 0$ reduces this geometry to the $\mathrm{AdS}$ one given in the previous section.

The metric (4.32) is a Schrödinger spacetime with $Z=0$ and the wrong sign. It is well known that this geometry can be regarded as a Lifshitz geometry with $Z=2$ after an appropriate compactification [31,32]. This can be easily seen by performing the completing square with respect to $d x^{-}$. The resulting metric is given by

$d s^{2}=\Delta_{\mathcal{O}}\left[-\frac{\left(d x^{+}\right)^{2}}{\gamma \tau^{4}}+\frac{d \tau^{2}+d \vec{x}^{2}}{\tau^{2}}+\gamma\left(d x^{-}+\frac{d x^{+}}{\gamma \tau^{2}}\right)^{2}\right]$.

By taking a compactification along the $x^{-}$-direction, this metric describes a Lifshitz spacetime [31,32].

\section{Light-cone reversal symmetry with the relativistic flow}

As another simple example, we consider a holographic geometry emerging from the nonrelativistic theory with the light-cone reversal symmetry $\left(x^{ \pm} \rightarrow-x^{ \pm}\right)$generated by the relativistic flow $(\bar{m}=0)$. This implies that the 2-point function of the normalized field given by (4.19) is invariant under $x_{12}^{ \pm} \rightarrow-x_{12}^{ \pm}$, so that we can write $G(u, v)=\hat{G}\left(u, v^{2}\right)$ with an unknown function $\hat{G}$. Therefore, we have
$G^{(n, 1)}(\overrightarrow{0})=0, \quad G^{(0,2)}(\overrightarrow{0})=2 \hat{G}^{(0,1)}(\overrightarrow{0})$,

$G^{(1,0)}(\overrightarrow{0})=-\frac{\Delta_{\mathcal{O}}}{2 D}$.

Setting $\eta=\frac{\tau^{2}}{2 D}$, we obtain

$d s^{2}=\Delta_{\mathcal{O}}\left[-\frac{2 D^{2} \hat{G}^{(0,1)}(\overrightarrow{0})}{\Delta_{\mathcal{O}}} \frac{\left(d x^{+}\right)^{2}}{\tau^{4}}+\frac{d \tau^{2}+2 d x^{+} d x^{-}+d \vec{x}^{2}}{\tau^{2}}\right]$.

This metric describes nothing but a Schrödinger spacetime with $\sigma^{2}:=\frac{2 D^{2} \hat{G}^{(0,1)}(\overrightarrow{0})}{\Delta_{\mathcal{O}}}$ studied in Ref. [18].

\section{Nonrelativistic deformation of a conformal primary}

As a more nontrivial example, we consider a nonrelativistic deformation of a conformal primary scalar operator in a relativistic CFT which preserve the property of the nonrelativistic conformal primary condition.

Let us consider a general CFT in Sec. III and deform the conformal primary scalar field $O_{0}(x)$ as

$$
O_{\epsilon}(x):=e^{\epsilon \partial^{2}}-O_{0}(x),
$$

where $\epsilon$ is a real deformation parameter. In order for the deformed operator to be well behaved, the parameter $\epsilon$ needs to be positive. This will turn out to be important to obtain a Schrödinger space-time with the correct sign.

Let us show that the deformed operator $O_{\epsilon}(x)$ is a conformal primary operator in the nonrelativistic conformal algebra. The conformal transformation of $O_{\epsilon}(x)$ is given by

$\delta_{\epsilon}^{\mathrm{conf}} O_{\epsilon}(x)=e^{\epsilon \partial_{-}^{2}} \delta^{\mathrm{conf}} O_{0}(x)=\delta^{\mathrm{conf}} O_{\epsilon}(x)+\delta_{\epsilon}^{\prime} O_{\epsilon}(x)$,

where $\delta^{\text {conf }}$ is given by (4.3) with (4.2), and

$$
\begin{aligned}
\delta_{\epsilon}^{\prime} O_{\epsilon}(x)= & 2 \epsilon\left[\left(2 b \cdot x-\lambda-\omega^{-+}\right) \partial_{-}^{2}-\omega^{i+} \partial_{i} \partial_{-}\right. \\
& +2\left(x \cdot \partial+\Delta_{\mathcal{O}}+1\right) b^{+} \partial_{-} \\
& \left.-2 x^{+} b \cdot \partial \partial_{-}+4 \epsilon b^{+} \partial_{-}^{3}\right] O_{\epsilon}(x) .
\end{aligned}
$$

$\delta_{\epsilon}^{\prime} O_{\epsilon}$ vanishes if and only if

$$
\omega^{+-}=\lambda, \quad \omega^{i+}=b^{i}=b^{+}=0 .
$$

The subalgebra with this parameter constraint is nothing but the Schrödinger algebra given in (4.5). In other words, the subalgebra commuting with the operator $e^{\epsilon \partial_{-}^{2}}$ becomes the Schrödinger algebra. Therefore the deformed operator $O_{\epsilon}(x)$ is a nonrelativistic conformal primary operator.

Hence we can apply the result in Sec. IVA to this deformed operator. The induced geometry obtained from 
the flowed operator of $O_{\epsilon}$ by the nonrelativistic flow equation (4.8) is given by (4.26), where the metric components are determined as a function of $\epsilon$.

An interesting situation happens when $\bar{m}=0$, where the flow equation becomes relativistic. In this case both CFT and the flow equation enjoy the light-cone reversal symmetry, so that the situation reduces to Sec. IV C, and the induced metric reduces to a Schrödinger one given by (4.36):

$d s^{2}=\Delta_{\mathcal{O}}\left[-\frac{2 D^{2} \hat{G}_{\epsilon}^{(0,1)}(\overrightarrow{0})}{\Delta_{\mathcal{O}}} \frac{\left(d x^{+}\right)^{2}}{\tau^{4}}+\frac{d \tau^{2}+2 d x^{+} d x^{-}+d \vec{x}^{2}}{\tau^{2}}\right]$,

where $\hat{G}_{\epsilon}^{(0,1)}$ describes the 2-point function of the normalized flowed field:

$$
\begin{aligned}
& \left\langle\sigma_{\epsilon}\left(x_{1} ; \eta_{1}\right) \sigma_{\epsilon}\left(x_{2} ; \eta_{2}\right)\right\rangle \\
& =\left(\frac{2 \sqrt{\eta_{1} \eta_{2}}}{\eta_{+}}\right)^{\Delta_{\mathcal{O}}} \hat{G}_{\epsilon}\left(\frac{2 x_{12}^{+} x_{12}^{-}+\left(\vec{x}_{12}\right)^{2}}{\eta_{+}},\left(\frac{x_{12}^{+}}{\eta_{+}}\right)^{2}\right) .
\end{aligned}
$$

In the current case, we can evaluate $\hat{G}_{\epsilon}^{(0,1)}(\overrightarrow{0})$. To this end let us investigate the transformation rule of the flowed field $\phi_{\epsilon}(x ; \eta)$ under the conformal transformation:

$$
\begin{aligned}
\delta_{\epsilon}^{\mathrm{conf}} \phi_{\epsilon}(x ; \eta) & =e^{\eta\left(\vec{\partial}^{2}+2 \partial_{+} \partial_{-}\right)+\epsilon \partial_{-}^{2}} \delta^{\mathrm{conf}} O(x) \\
& =\delta^{\mathrm{conf}} \phi_{\epsilon}(x ; \eta)+\delta_{\epsilon}^{\prime} \phi_{\epsilon}(x ; \eta)
\end{aligned}
$$

where

$$
\begin{aligned}
\delta_{\epsilon}^{\prime} \phi_{\epsilon}(x ; \eta): & =\left[2 \eta\left\{(2 b \cdot x-\lambda) \partial_{\eta}-\left(d-1-2 \Delta_{\mathcal{O}}\right) b \cdot \partial\right\}\right. \\
& +2 \epsilon\left\{\left(2 b \cdot x-\lambda-\omega^{-+}\right) \partial_{-}^{2}\right. \\
& \left.+2\left(x \cdot \partial+\Delta_{\mathcal{O}}+1\right) b^{+} \partial_{-}-\omega^{i+} \partial_{i} \partial_{-}-2 x^{+} \partial_{-} b \cdot \partial\right\} \\
& \left.+4 \eta^{2} b \cdot \partial \partial_{\eta}+8 \epsilon \eta b^{+} \partial_{-} \partial_{\eta}+8 \epsilon^{2} b^{+} \partial_{-}^{3}\right] \phi_{\epsilon}(x ; \eta) .
\end{aligned}
$$

If we restrict the conformal transformation to the Schrödinger one, (4.40), then the terms dependent on $\epsilon$ drop out and we have (4.11) with $\bar{m}=0$. Since the 2-point function is invariant under the rotation parametrized by $\omega^{i+}$, which is outside the Schrödinger algebra, we find

$$
\left(x^{-} \partial_{i}-x^{i} \partial_{+}+4 \epsilon \partial_{-} \partial_{i}\right) \hat{G}_{\epsilon}\left(\frac{\vec{x}^{2}+2 x^{+} x^{-}}{\eta}, \frac{\left(x^{+}\right)^{2}}{\eta^{2}}\right)=0,
$$

which leads to

$$
\hat{G}_{\epsilon}^{(0,1)}(u, v)=8 \epsilon \hat{G}_{\epsilon}^{(2,0)}(u, v) .
$$

On the other hand, (4.25) gives
$\hat{G}_{\epsilon}^{(2,0)}(\overrightarrow{0})=-\frac{\Delta_{\mathcal{O}}+1}{2(D+2)} \hat{G}_{\epsilon}^{(1,0)}(\overrightarrow{0})=\frac{\Delta_{\mathcal{O}}\left(\Delta_{\mathcal{O}}+1\right)}{4 D(D+2)}$.

Therefore we obtain

$$
\hat{G}_{\epsilon}^{(0,1)}(\overrightarrow{0})=2 \epsilon \frac{\Delta_{\mathcal{O}}\left(\Delta_{\mathcal{O}}+1\right)}{D(D+2)} .
$$

Finally the induced line element is obtained as

$$
\begin{aligned}
d s^{2}= & \Delta_{\mathcal{O}}\left[-\epsilon \frac{4\left(\Delta_{\mathcal{O}}+1\right) D}{D+2} \frac{\left(d x^{+}\right)^{2}}{\tau^{4}}\right. \\
& \left.+\frac{d \tau^{2}+2 d x^{+} d x^{-}+d \vec{x}^{2}}{\tau^{2}}\right]
\end{aligned}
$$

Since the parameter $\epsilon$ is positive, this is a Schrödinger space-time with the correct sign.

This result is in fact guaranteed by the symmetry. Let us restrict the argument of conformal symmetry in the previous section to that of the nonrelativistic one. Then the normalized field $\sigma_{\epsilon}(x ; \eta)$ transforms under the Schrödinger transformation as

$$
\delta_{\epsilon}^{S} \sigma_{\epsilon}(x ; \eta)=\delta_{S}^{\mathrm{diff}} \sigma_{\epsilon}(x ; \eta)+\delta^{\mathrm{extra}} \sigma_{\epsilon}(x ; \eta),
$$

where $\delta_{S}^{\text {diff }}$ generates isometries of the Schrödinger spacetime as

$$
\begin{aligned}
\delta_{s}^{\text {diff }} \sigma_{\epsilon}(x ; \eta) & =-\bar{\delta}^{s} x^{A} \partial_{A} \sigma_{\epsilon}(x ; \eta), \\
\bar{\delta}^{s} x^{i} & =a^{i}+\omega^{i}{ }_{j} x^{j}-v^{i} x^{+}+\lambda x^{i}-2 b x^{+} x^{i}, \\
\bar{\delta}^{s} x^{+} & =a^{+}+2 \lambda x^{+}-2 b\left(x^{+}\right)^{2}, \\
\bar{\delta}^{s} x^{-} & =\mu+v^{i} x^{i}+b\left(\vec{x}^{2}+\tau^{2}\right), \quad \bar{\delta}^{s} \tau=\left(2 b x^{+}-\lambda\right) \tau,
\end{aligned}
$$

while the extra contribution becomes

$\delta^{\mathrm{extra}} \sigma_{\epsilon}(x ; \eta)=4 b \eta^{2} \partial_{-}\left(\partial_{\eta}+\frac{\Delta_{\mathcal{O}}+2}{2 \eta}\right) \sigma_{\epsilon}(x ; \eta)$.

From this it follows that

$$
\begin{aligned}
& \delta^{\text {extra }}\left\langle\sigma_{\epsilon}\left(x_{1} ; \eta_{1}\right) \sigma_{\epsilon}\left(x_{2} ; \eta_{2}\right)\right\rangle \\
& =-8 b x_{12}^{+} x_{12}^{2} \frac{\left(\eta_{1}-\eta_{2}\right)}{\eta_{+}}\left(\frac{2 \sqrt{\eta_{1} \eta_{2}}}{\eta_{+}}\right)^{\Delta_{\mathcal{O}}} \hat{G}_{\epsilon}^{(2,0)}\left(\frac{x_{12}^{2}}{\eta_{+}}, \frac{\left(x_{12}^{+}\right)^{2}}{\eta_{+}^{2}}\right) .
\end{aligned}
$$

Therefore, we have $\delta^{\text {extra }} g_{A B}(z)=0$, which implies $\delta_{S}^{\text {diff }} g_{A B}(z)=0$. This shows that the resulting induced metric is invariant under the transformations forming the Schrödinger algebra, which requires the geometry to be a Schrödinger spacetime. 


\section{E. Mass eigenvector}

Finally we consider the case where a conformal primary scalar in a nonrelativistic CFT becomes a mass eigenvector such that

$$
\left[M, O_{m}\right]=m O_{m}, \quad M=i \partial_{-}
$$

with a mass parameter $m$, and the transformation of the operator $O_{m}$ is given by (4.6). Then the 2-point function of this primary operator given by (4.7) is now more constrained as $[13,17]$

$\left\langle O_{m}\left(\vec{x}_{1}, x_{1}^{+}, x_{1}^{-}\right) O_{m}\left(\vec{x}_{2}, x_{2}^{+}, x_{2}^{-}\right)^{\dagger}\right\rangle=\frac{C \exp \left[\operatorname{im}\left(x_{12}^{-}+\frac{\vec{x}_{12}^{2}}{2 x_{12}^{+}}\right)\right]}{\left(x_{12}^{+}\right)^{\Delta_{\mathcal{O}}}}$

with a constant $C$.

Smearing by the flow equation (4.8) does not break the property of the operator as an eigenvector for the mass operator, so the 2-point function of the flowed operator, which we denote by $\phi_{m}$, further reduces from (4.18) to

$$
\begin{aligned}
& \left\langle\phi_{m}\left(x_{1} ; \eta_{1}\right) \phi_{m}^{\dagger}\left(x_{2} ; \eta_{2}\right)\right\rangle \\
& \quad=\frac{\exp \left[i m\left(x_{12}^{-}+\frac{\left(\vec{x}_{12}\right)^{2}}{2\left(x_{12}^{+} 2 i \bar{m} \eta_{+}\right)}\right)\right]}{\eta_{+}^{\Delta_{\mathcal{O}}}} F_{m}\left(\frac{x_{12}^{+}}{\eta_{+}} ; 1\right) .
\end{aligned}
$$

It is important to note the introduction of nonzero $\bar{m}$ in the flow equation (4.8) is needed to resolve the contact singularity in the time $\left(x^{+}\right)$direction. Therefore the function $G$ in Sec. IVA reduces to

$$
\begin{aligned}
G & \left(\frac{2\left(x^{+}+2 i \bar{m} \eta_{+}\right) x^{-}+\vec{x}^{2}}{\eta_{+}}, \frac{x^{+}}{\eta_{+}}\right) \\
\quad & \exp \left[i m\left(x^{-}+\frac{\vec{x}^{2}}{2\left(x^{+}+2 i \bar{m} \eta_{+}\right)}\right)\right] G_{m}\left(\frac{x^{+}}{\eta_{+}}\right) .
\end{aligned}
$$

This suggests that the induced line element (4.26) becomes

$$
\begin{aligned}
d s^{2}= & \frac{\Delta_{\mathcal{O}}}{4 \eta^{2}} d \eta^{2}+\frac{-G_{m}^{\prime \prime}(0)}{4 \eta^{2}}\left(d x^{+}\right)^{2} \\
& +\frac{-i m G_{m}^{\prime}(0)}{\eta} d x^{+} d x^{-}+m^{2}\left(d x^{-}\right)^{2}+\frac{-m}{4 \bar{m} \eta} d \vec{x}^{2} .
\end{aligned}
$$

The coefficients $G_{m}^{\prime}(0)$ and $G_{m}^{\prime \prime}(0)$ are determined from (4.24) and (4.25) as follows.

$$
\begin{aligned}
G_{m}^{\prime}(0) & =-\frac{\Delta_{\mathcal{O}}+\frac{m(d-1)}{2 \bar{m}}}{2 i(m+\bar{m})} \\
G_{m}^{\prime \prime}(0) & =\frac{-\left(\Delta_{\mathcal{O}}+1+\frac{m(d-1)}{2 \bar{m}}\right) G_{m}^{\prime}(0)-\frac{i m(d-1)}{4 \bar{m}^{2}}}{2 i(m+\bar{m})}
\end{aligned}
$$

This geometry becomes a Lifshitz one with $Z=2$ after an appropriate compactification in some parameter region. To realize this, we tune a parameter in the nonrelativistic flow as $\bar{m}=-c m$ with $c>0$. Then, by setting

$$
\eta=\frac{-m}{4 \Delta_{\mathcal{O}} \bar{m}} \tau^{2}=\frac{1}{4 c \Delta_{\mathcal{O}}} \tau^{2}
$$

the above metric becomes

$d s^{2}=\Delta_{\mathcal{O}}\left(c_{1} \frac{\left(d x^{+}\right)^{2}}{\tau^{4}}+\frac{d \tau^{2}+d \vec{x}^{2}}{\tau^{2}}\right)+m^{2}\left(d x^{-}+c_{2} \frac{d x^{+}}{\tau^{2}}\right)^{2}$,

where $c_{1}$ and $c_{2}$ are given by

$c_{1}=\Delta_{\mathcal{O}} \frac{c^{2}\left(2 \Delta_{\mathcal{O}}-d+1\right)+(d-1)(1-c)^{2}}{2(1-c)^{2}}$,

$c_{2}=\frac{\left.\Delta_{\mathcal{O}}\left(2 c \Delta_{\mathcal{O}}-d+1\right)\right)}{2 m^{2}(1-c)}$.

Thus, $c_{1}$ has to be negative so that the metric (4.58) may be regarded as a Lifshitz spacetime with $Z=2$. This is realized in a region specified by

$$
(0<) \Delta_{\mathcal{O}}<\left(\frac{d-1}{2}\right) \frac{2 c-1}{c^{2}},
$$

where the upper bound becomes maximum as $\Delta_{\mathcal{O}}<(d-1) / 2$ at $c=1$.

\section{HOLOGRAPHIC GEOMETRY FROM A LIFSHITZ-TYPE SCALE-INVARIANT THEORY}

In this section, we consider a Lifshitz-type scale-invariant field theory, which is not necessarily conformally invariant. There exists a primary scalar operator $O_{Z}(t, \vec{x})$, which transforms under the dilatation as ${ }^{10}$

$$
O_{Z}\left(\Lambda \vec{x}, \Lambda^{Z} t\right)=\Lambda^{-\Delta_{\mathcal{O}}} O_{Z}(\vec{x}, t)
$$

with a scaling factor $Z$. Using the invariance under the translation and the special rotation, the 2-point function of this scalar operator is constrained as

\footnotetext{
${ }^{10}$ The infinitesimal transformation is $\delta_{\lambda} O_{Z}(\vec{x}, t)=-\lambda\left(\Delta_{\mathcal{O}}+\right.$ $\left.x_{i} \partial_{i}+Z t \partial_{t}\right) O_{Z}(\vec{x}, t)$, where $\Lambda=e^{\lambda}$.
} 


$$
\left\langle O_{Z}\left(\vec{x}_{1}, t_{1}\right) O_{Z}\left(\vec{x}_{2}, t_{2}\right)\right\rangle=\frac{f_{Z}\left(\frac{t_{12}^{2 / Z}}{\vec{x}_{12}^{2}}\right)}{\left(\vec{x}_{12}^{2}\right)^{\Delta_{\mathcal{O}}}}
$$

where $f_{Z}$ is a function characterized by the original theory. We assumed it to be smooth in terms of the time and invariant under the time reversal. ${ }^{11}$

A flow equation in this case should be a diffusion type and compatible with the scaling relation. We can easily find out such a flow equation as

$$
\begin{aligned}
\frac{\partial \phi_{Z}(\vec{x}, t ; \eta)}{\partial \eta} & =\left(\vec{\partial}^{2}+a Z \eta^{Z-1} \partial_{t}^{2}\right) \phi_{Z}(\vec{x}, t ; \eta) \\
\phi_{Z}(\vec{x}, t ; 0) & =O_{Z}(\vec{x}, t)
\end{aligned}
$$

where we here introduced a positive parameter $a$. We call this flow a Lifshitz flow. The formal solution is given by

$$
\phi_{Z}(\vec{x}, t ; \eta)=e^{\eta \vec{\partial}^{2}+a \eta^{Z} \partial_{t}^{2}} O_{Z}(\vec{x}, t)
$$

The 2-point function of the flowed field $\phi_{Z}$ can be written as

$$
\begin{aligned}
& \left\langle\phi_{Z}\left(\vec{x}_{1}, t_{1} ; \eta_{1}\right) \phi_{Z}\left(\vec{x}_{2}, t_{2} ; \eta_{2}\right)\right\rangle \\
& \quad=e^{\eta_{1} \vec{\partial}^{2}+a \eta_{1}^{Z} \partial_{t}^{2}+\eta_{2} \vec{\partial}^{\prime 2}+a \eta_{2}^{Z} \partial_{t}{ }^{\prime 2}}\left\langle O_{Z}\left(\vec{x}_{1}, t_{1}\right) O_{Z}\left(\vec{x}_{2}, t_{2}\right)\right\rangle \\
& \quad=e^{\eta_{+} \vec{\partial}^{2}+a \eta_{Z+} \partial_{t}^{2}}\left\langle O_{Z}\left(\vec{x}_{12}, t_{12}\right) O_{Z}(\overrightarrow{0}, 0)\right\rangle
\end{aligned}
$$

where we used (5.2) and set $\eta_{Z_{+}}:=\eta_{1}^{Z}+\eta_{2}^{Z}$. Taking into account (5.2) we can denote this function by $F_{Z}\left(\vec{x}_{12}^{2}, t_{12}^{2} ; 2^{Z-1} \eta_{Z+}, \eta_{+}\right)$. Here we used the assumption that the function $f$ in (5.2) is smooth with respect to the time.

By using the scaling relation (5.1) the function $F_{Z}$ satisfies

$$
\begin{gathered}
F_{Z}\left(\Lambda^{2} x^{2}, \Lambda^{2 Z} t^{2} ; \Lambda^{2 Z} 2^{Z-1} \eta_{Z+}, \Lambda^{2} \eta_{+}\right) \\
=\Lambda^{-2 \Delta_{\mathcal{O}}} F_{Z}\left(x^{2}, t^{2} ; 2^{Z-1} \eta_{Z+}, \eta_{+}\right)
\end{gathered}
$$

Choosing $\Lambda=1 / \eta_{+}^{\frac{1}{2}}$ we find

$F_{Z}\left(\vec{x}^{2}, t^{2} ; \eta_{Z+}, \eta_{+}\right)=\frac{1}{\eta_{+}^{\Delta_{\mathcal{O}}}} F_{Z}\left(\frac{\vec{x}^{2}}{\eta_{+}}, \frac{t^{2}}{\eta_{+}^{Z}} ; \frac{2^{Z-1} \eta_{Z+}}{\eta_{+}^{Z}}, 1\right)$,

from which the normalized flowed field is given by

$$
\sigma_{Z}(\vec{x}, t ; \eta)=\frac{(2 \eta)^{\Delta_{\mathcal{O}} / 2}}{\sqrt{F_{Z}(0,0 ; 1,1)}} \phi_{Z}(\vec{x}, t ; \eta)
$$

so that

\footnotetext{
${ }^{11}$ If we do not assume this, then terms such as $d \eta d x^{+}$appear in the induced metric.
}

$$
\begin{aligned}
& \left\langle\sigma_{Z}\left(\vec{x}_{1}, t_{1} ; \eta_{1}\right) \sigma_{Z}\left(\vec{x}_{2}, t_{2} ; \eta_{2}\right)\right\rangle \\
& =\left(\frac{2 \sqrt{\eta_{1} \eta_{2}}}{\eta_{+}}\right)^{\Delta_{\mathcal{O}}} G_{Z}\left(\frac{\vec{x}_{12}^{2}}{\eta_{+}}, \frac{t_{12}^{2}}{\eta_{+}^{Z}}, \frac{2^{Z-1} \eta_{Z+}}{\eta_{+}^{Z}}\right),
\end{aligned}
$$

where $G_{Z}\left(x_{1}, x_{2}, x_{3}\right):=F_{Z}\left(x_{1}, x_{2} ; x_{3}, 1\right) / F_{Z}(0,0 ; 1,1)$. This function $G_{Z}$ is constrained by the flow equation:

$$
\begin{aligned}
& \partial_{\eta_{1}}\left\langle\phi_{Z}\left(\vec{x}_{1}, t_{1} ; \eta_{1}\right) \phi_{Z}\left(\vec{x}_{2}, t_{2} ; \eta_{2}\right)\right\rangle \\
& \quad=\left(\vec{\partial}^{2}+a Z \eta_{1}^{Z-1} \partial_{t}^{2}\right)\left\langle\phi_{Z}\left(\vec{x}_{1}, t_{1} ; \eta_{1}\right) \phi_{Z}\left(\vec{x}_{2}, t_{2} ; \eta_{2}\right)\right\rangle
\end{aligned}
$$

which leads to

$$
\begin{aligned}
-\Delta_{\mathcal{O}}= & 2(d-1) G_{Z}^{(1,0,0)}(0,0,1) \\
& +a Z 2^{2-Z} G_{Z}^{(0,1,0)}(0,0,1), \\
G_{Z}^{(0,0,1)}(0,0,1)= & \frac{a}{2^{Z-2}} G_{Z}^{(0,1,0)}(0,0,1) .
\end{aligned}
$$

Nonzero components of the induced metric are calculated as

$$
\begin{array}{r}
g_{\eta \eta}(z)=\frac{\Delta_{\mathcal{O}}}{4 \eta^{2}}-\frac{Z(Z-1)}{4 \eta^{2}} G_{Z}^{(0,0,1)}(0,0,1), \\
g_{t t}(z)=\frac{-2}{(2 \eta)^{Z}} G_{Z}^{(0,1,0)}(0,0,1), \\
g_{i j}(z)=\frac{-\delta_{i j}}{\eta} G_{Z}^{(1,0,0)}(0,0,1) .
\end{array}
$$

Therefore

$$
\begin{aligned}
d s^{2}= & \left(\frac{\Delta_{\mathcal{O}}}{4 \eta^{2}}-\frac{Z(Z-1)}{4 \eta^{2}} G_{Z}^{(0,0,1)}(0,0,1)\right) d \eta^{2} \\
& +\frac{-2}{(2 \eta)^{Z}} G_{Z}^{(0,1,0)}(0,0,1)(d t)^{2} \\
& +\frac{-\delta_{i j} G_{Z}^{(1,0,0)}(0,0,1)}{\eta} d x^{i} d x^{j} \\
= & \left(\Delta_{\mathcal{O}}-a Z(Z-1) 2^{2-Z} G_{Z}^{(0,1,0)}(0,0,1)\right) \\
& \times \frac{d \tau^{2}+d \vec{x}^{2}}{\tau^{2}}-\frac{2 G_{Z}^{(0,1,0)}(0,0,1)}{(2 \alpha)^{Z}} \frac{(d t)^{2}}{\tau^{2 Z}}
\end{aligned}
$$

where we set $\eta:=\alpha \tau^{2}$ with

$\alpha=\frac{\Delta_{\mathcal{O}}+a Z 2^{2-Z} G_{Z}^{(0,1,0)}(0,0,1)}{2(d-1)\left(\Delta_{\mathcal{O}}-a Z(Z-1) 2^{2-Z} G_{Z}^{(0,1,0)}(0,0,1)\right)}$.

This describes a Lifshitz geometry with a general dynamical exponent $Z$. 


\section{CONCLUSION AND DISCUSSION}

We have extended the construction of holographic geometries by means of the flow equation approach to nonrelativistic scale invariant theories. After reviewing the construction of the AdS space by using a general CFT both in the Euclidean and the Lorentzian space, we moved on to the construction of holographic geometries of a nonrelativistic CFT and a nonrelativistic flow equation. As a result we have obtained a hybrid geometry of both Schrödinger and Lifshitz geometries as a general holographic spacetime in this framework. Applying this result to specific nonrelativistic models, we have reproduced a Schrödinger geometry and a Lifshitz one with $Z=2$. We have also reproduced a Lifshitz geometry with a general dynamical exponent by smearing an operator of a Lifshitz theory with a suitable modification of the flow equation.

It would be an interesting problem to realize the seemingly new geometry we called the NR hybrid geometry in Sec. IV A as a solution of a certain bulk theory. Such a bulk theory may be realized as a usual gravitational theory coupling to matter fields in a similar way with Lifshitz and Schrödinger geometries (see [33] for a review and references therein).

Although the flow field approach seems to provide new perspective to investigate the holography, there are still gaps to fill in between them. One of them is the relationship between flowed operators in a CFT and bulk operators appearing in the standard AdS/CFT correspondence. It may be clear that they are conceptually different, because the 2-point function of a flowed operator does not have contact singularity, while that of a bulk local field has. Indeed there is a standard construction of bulk operators from a Lorentzian CFT known as the Hamilton-Kabat-Lifshitz-Low construction [34], where bulk operators are obtained by convoluting CFT operators with a certain smearing function. Their striking result is that such a smearing is done over the causally disconnected region to obtain a bulk operator in even dimensional Poincaré AdS, while smearing is done all over the region for odd dimensional one. In Sec. III B and Appendix B we smeared a CFT primary operator in Lorentzian flows. In both cases smearing region is basically done all over the space. It is important to understand how smearing encodes the causality in the Lorentzian space in the flow equation approach.

In relation to the above, it is also important to investigate the correspondence of excited states between the bulk and boundary in the flow field approach. There are orthodox ways to study bulk geometries corresponding to an excited state (for example [35]), while there is a proposal how to compute a back-reacted geometry by an excited state in the flow field approach [36]. It is intriguing to see whether a resulting induced geometry have desired properties and match one constructed by a different approach.

We hope to come back to these issues in the near future.

\section{ACKNOWLEDGMENTS}

We are very grateful to Yoshihiko Abe for valuable comments and useful discussions. The authors thank the Yukawa Institute for Theoretical Physics at Kyoto University. We would like to thank Janos Balog for his valuable comments on the first version of this paper. Discussions during the workshop YITP-T-18-04 "New Frontiers in String Theory 2018" were useful to complete this work. S. A. is supported in part by the Grant-in-Aid of the Japanese Ministry of Education, Sciences and Technology, Sports and Culture (MEXT) for Scientific Research (No. JP16H03978, No. JP18H05236), by a priority issue (Elucidation of the fundamental laws and evolution of the universe) to be tackled by using Post "K" Computer, and by Joint Institute for Computational Fundamental Science (JICFuS). The work of K. Y. was supported by the Supporting Program for Interaction-based Initiative Team Studies (SPIRITS) from Kyoto University, a JSPS Grant-in-Aid for Scientific Research (B) No. 18H01214. This work is also supported in part by the JSPS Japan-Russia Research Cooperative Program.

\section{APPENDIX A: CONFORMAL, SCHRÖDINGER AND LIFSHITZ ALGEBRAS}

In this Appendix we shall describe how the Schrödinger and Lifshitz algebras are embedded into the conformal algebra $\mathfrak{g} \mathfrak{o}(2, D)$, and present the transformation laws under the Schrödinger and Lifshitz symmetries.

\section{Conformal algebra $\mathfrak{g} \mathfrak{o}(2, D)$}

Let us begin with a conformal algebra $\mathfrak{g} \mathfrak{o}(2, D)$ in $D$ dimensional Minkowski spacetime, which is generated by antisymmetric matrices $M_{A B}$ satisfying

$\left[M_{A B}, M_{C D}\right]=i g_{A C} M_{B D}-i g_{B C} M_{A D}-i g_{A D} M_{B C}+i g_{B D} M_{A C}$.

Here the indices are the metric components are given by

$$
\begin{aligned}
A, B, \ldots & =-1,0,1, \ldots, D, \\
-g_{-1-1} & =-g_{00}=g_{11}=\cdots=g_{D D}=1 .
\end{aligned}
$$

In the following, it is helpful to introduce the light-cone coordinates:

$$
x^{\tilde{ \pm}}=\frac{1}{\sqrt{2}}\left(x^{D} \pm x^{-1}\right) .
$$

Then the components of $M_{A B}$ can be presented in terms of the conformal basis $P_{\mu}$ (translation), $M_{\mu \nu}$ (Lorentz rotation), $D$ (dilatation) and $K_{\mu}$ (special conformal) as follows: 


$$
\left(M_{A B}\right)=\begin{gathered}
\nu \\
\mu \\
\tilde{+} \\
\sim
\end{gathered}\left(\begin{array}{ccc}
M_{\mu \nu} & P_{\mu} & K_{\mu} \\
-P_{\nu} & 0 & -D \\
-K_{\nu} & D & 0
\end{array}\right) .
$$

Now the commutation relation in (A1) can be decomposed into the following standard form:

$$
\begin{gathered}
{\left[M_{\mu \nu}, M_{\rho \delta}\right]=i g_{\mu \rho} M_{\nu \delta}-i g_{\nu \rho} M_{\mu \delta}-i g_{\mu \delta} M_{\nu \rho}+i g_{\nu \delta} M_{\mu \rho}} \\
{\left[M_{\mu \nu}, P_{\rho}\right]=i g_{\mu \rho} P_{\nu}-i g_{\nu \rho} P_{\mu}} \\
{\left[M_{\mu \nu}, K_{\rho}\right]=i g_{\mu \rho} K_{\nu}-i g_{\nu \rho} K_{\mu},} \\
{\left[K_{\mu}, P_{\nu}\right]=i g_{\mu \nu} D+i M_{\mu \nu},} \\
{\left[D, M_{\mu \nu}\right]=0, \quad\left[D, P_{\nu}\right]=i P_{\nu}, \quad\left[D, K_{\nu}\right]=-i K_{\nu} .}
\end{gathered}
$$

\section{Schrödinger algebra from $\mathfrak{s} \mathfrak{v}(2, D)$}

To see the Schrödinger algebra as a subalgebra of $\mathfrak{s} \mathfrak{o}(2, D)$, it is useful to introduce another couple of the light-cone coordinates $x^{ \pm}$with $x^{0}$ and $x^{D-1}$ :

$$
x^{ \pm}=\frac{1}{\sqrt{2}}\left(x^{D-1} \pm x^{0}\right)
$$

Then the generators of $\mathfrak{s} \mathfrak{o}(2, D)$ can be displayed as

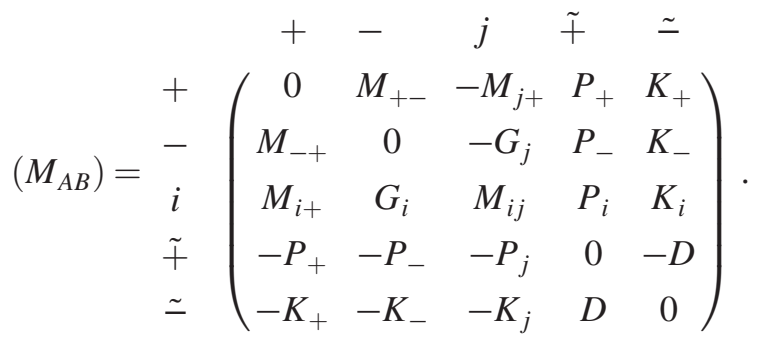

It is significant to notice that one can find out a subalgebra by dropping the generators $M_{i+}, K_{i}$ and $K_{+}$. By introducing the following notation

$H \equiv P_{+}, \quad M \equiv P_{-}, \quad K \equiv K_{-}, \quad \mathcal{D}=D+M_{-+}$,

the subalgebra is given by

$$
\begin{aligned}
{\left[M_{i j}, M_{k l}\right] } & =i g_{i k} M_{j l}-i g_{j k} M_{i l}-i g_{i l} M_{j k}+i g_{j l} M_{i k}, \\
{\left[M_{i j}, P_{k}\right] } & =i g_{i k} P_{j}-i g_{j k} P_{i}, \quad\left[M_{i j}, G_{k}\right]=i g_{i k} G_{j}-i g_{j k} G_{i} \\
{\left[G_{i}, P_{j}\right] } & =i g_{i j} M, \quad\left[H, G_{i}\right]=i P_{i}, \quad[H, K]=-i \mathcal{D}, \\
{\left[\mathcal{D}, P_{j}\right] } & =i P_{j}, \quad\left[\mathcal{D}, G_{j}\right]=-i G_{j}, \\
{[\mathcal{D}, H] } & =2 i H, \quad[\mathcal{D}, K]=-2 i K,
\end{aligned}
$$

and the other commutation relations vanish. This is nothing but the Schrödinger algebra. ${ }^{12}$ This algebra is composed of $H$ (time translation), $P_{i}$ (spatial translation), $M_{i j}$ (spatial rotation), $G_{i}$ (Galilean boost), $\mathcal{D}$ (anisotropic dilatation) $K$ (special conformal) and $M$ (mass operator).

As one can notice from the commutation relations in (A11), the scale transformation associated with $\mathcal{D}$ is anisotropic like

$t \rightarrow \Lambda^{2} t, \quad x^{i} \rightarrow \Lambda x^{i} \quad(\Lambda:$ a real constant parameter $)$.

This is a characteristic of the Schrödinger algebra.

\section{Lifshitz algebra from Schrödinger algebra}

The Lifshitz algebra is embedded as a subalgebra of the Schrödinger algebra when the dynamical exponent is two. This embedding can be seen by dropping off the generators $G_{i}, K$ and $M$ from the Schrödinger algebra (A11). The resulting commutation relations are given by

$$
\begin{aligned}
{\left[M_{i j}, M_{k l}\right] } & =i g_{i k} M_{j l}-i g_{j k} M_{i l}-i g_{i l} M_{j k}+i g_{j l} M_{i k}, \\
{\left[M_{i j}, P_{k}\right] } & =i g_{i k} P_{j}-i g_{j k} P_{i}, \\
{\left[\mathcal{D}, P_{j}\right] } & =i P_{j}, \quad[\mathcal{D}, H]=2 i H .
\end{aligned}
$$

In total, this algebra is composed of $H$ (time translation), $P_{i}$ (spatial translation), $M_{i j}$ (spatial rotation) and $\mathcal{D}$ (anisotropic dilatation). To recover the algebra with an arbitrary value of the dynamical exponent $Z$, the commutation relation involving the dilatation and Hamiltonian should be modified as

$$
[\mathcal{D}, H]=Z i H .
$$

\section{APPENDIX B: LORENTZ NONINVARIANT FLOW EQUATION}

In this Appendix we present a different method to flow a primary operator in Lorentzian CFT. Although this approach breaks the manifest Lorentz invariance, it has a virtue to obtain a well-defined flowed operator in the Lorentzian space.

The method is to introduce another flow parameter $\eta_{t}$ specially for the time direction as follows.

$$
\frac{\partial}{\partial \eta_{t}} \phi_{0}=-\partial_{t}^{2} \phi_{0}, \quad \frac{\partial}{\partial \eta} \phi_{0}=\sum_{i} \partial_{i}^{2} \phi_{0}
$$

\footnotetext{
${ }^{12}$ This is the Schrödinger algebra with the dynamical critical exponent $z_{c}=2$. One may consider an arbitrary value of $z_{c}$. But except $z_{c} \neq 2$ (and 1 ), the special conformal generator $K$ must be excluded so as to close the algebra.
} 
If the flow parameters are in the region that

$$
\eta_{t}<0, \quad \eta>0
$$

then the flow equation has a well-defined solution

$$
\begin{aligned}
\phi_{0}\left(x ; \eta_{t}, \eta\right) & =e^{-\eta_{t} \partial_{t}^{2}+\eta \partial_{i}^{2}} O_{0}(x) \\
& =\int d^{D} x^{\prime} K_{0}\left(x, x^{\prime} ; \eta_{t}, \eta\right) O_{0}\left(x^{\prime}\right)
\end{aligned}
$$

where

$$
K_{0}\left(x, x^{\prime} ; \eta_{t}, \eta\right)=\frac{e^{\frac{\left(t-t^{\prime}\right)^{2}}{4 \eta_{t}}}}{\left(4 \pi \eta_{t}\right)^{\frac{1}{2}}} \frac{e^{-\frac{\left(x^{i}-x^{\prime i}\right)^{2}}{4 \eta}}}{(4 \pi \eta)^{\frac{D-1}{2}}}
$$

Then the two point correlation function of the flowed operator is written as

$$
\begin{aligned}
& \left\langle\phi_{0}\left(x_{1} ; \eta_{t 1}, \eta_{1}\right) \phi_{0}\left(x_{2} ; \eta_{t 2}, \eta_{2}\right)\right\rangle \\
& =e^{-\eta_{t} \partial_{t}^{2}+\eta \partial_{i}^{2}-\eta_{t}^{\prime} \partial_{t}^{\prime 2}+\eta^{\prime} \partial_{i}{ }^{\prime 2}} f_{0}\left(x_{12}^{2}\right) \\
& =e^{-\eta_{t+} \partial_{t}^{2}+\eta_{+} \partial_{i}^{2}} f_{0}\left(x_{12}^{2}\right),
\end{aligned}
$$

where we used the notation in the main text. This is a function of $t_{12}^{2}, \vec{x}_{12}^{2}, \eta_{t+}, \eta_{+}$, which we denote by $F_{0}\left(t_{12}^{2}, \vec{x}_{12}^{2} ; \eta_{t+}, \eta_{+}\right)$. By using the scaling relation we find

$$
F_{0}\left(t^{2}, \vec{x}^{2} ; \eta_{t}, \eta\right)=\frac{1}{\eta^{\Delta_{\mathcal{O}}}} F_{0}\left(\frac{t^{2}}{\eta}, \frac{\vec{x}^{2}}{\eta} ; \frac{\eta_{t}}{\eta}, 1\right) .
$$

In particular

$$
\begin{aligned}
\left\langle\phi_{0}\left(x ; \eta_{t}, \eta\right)^{2}\right\rangle & =F_{0}\left(0,0 ; 2 \eta_{t}, 2 \eta\right) \\
& =\frac{1}{(2 \eta)^{\Delta_{\mathcal{O}}}} F_{0}\left(0,0 ; \frac{\eta_{t}}{\eta}, 1\right) .
\end{aligned}
$$

Furthermore we have

$$
\begin{gathered}
F_{0}^{(1,0,0,0)}\left(\frac{t^{2}}{\eta}, \frac{\vec{x}^{2}}{\eta} ; \frac{\eta_{t}}{\eta}, 1\right) \\
=(-1) \frac{\eta^{\Delta_{\mathcal{O}}}}{\eta} e^{-\eta_{t} \partial_{t}^{2}+\eta \partial_{i}^{2}} f_{0}^{\prime}\left(x_{12}^{2}\right) \\
=-F_{0}^{(0,1,0,0)}\left(\frac{t^{2}}{\eta}, \frac{\vec{x}^{2}}{\eta} ; \frac{\eta_{t}}{\eta}, 1\right) .
\end{gathered}
$$

Therefore the two point correlation function of the normalized field is given by

$$
\begin{aligned}
& \left\langle\sigma_{0}\left(x_{1} ; \eta_{t 1}, \eta_{1}\right) \sigma_{0}\left(x_{2} ; \eta_{t 2}, \eta_{2}\right)\right\rangle \\
& =\left(\frac{\sqrt{4 \eta_{1} \eta_{2}}}{\eta_{+}}\right)^{\Delta_{\mathcal{O}}} \frac{F_{0}\left(\frac{t_{12}^{2}}{\eta_{+}}, \frac{\vec{x}_{12}^{2}}{\eta_{+}} ; \frac{\eta_{t+}}{\eta_{+}}, 1\right)}{\sqrt{F_{0}\left(0,0 ; \frac{\eta_{t 1}}{\eta_{1}}, 1\right) F_{0}\left(0,0 ; \frac{\eta_{t 2}}{\eta_{2}}, 1\right)}} .
\end{aligned}
$$

The right-hand side is convergent and smooth for $\eta_{t 1}$, $\eta_{t 2}<0, \eta_{1}, \eta_{2}>0$. Therefore we perform the analytic continuation in terms of $\eta_{t 1}, \eta_{t 2}$ from the negative region to the positive value with $\eta_{t} \rightarrow \eta, \eta_{t 2} \rightarrow \eta_{2}$. Then the righthand side yields a smooth function of $\frac{t_{12}^{2}}{\eta_{+}}$and $\frac{\vec{x}_{12}^{2}}{\eta_{+}}$, which we denote as $\left(\frac{\sqrt{4 \eta_{1} \eta_{2}}}{\eta_{+}}\right)^{\Delta_{\mathcal{O}}} G_{0}\left(\frac{t_{12}^{2}}{\eta_{+}}, \frac{\vec{x}_{12}^{2}}{\eta_{+}}\right)$with $G_{0}(\overrightarrow{0})=1$. We thus obtain

$\left\langle\sigma_{0}\left(x_{1} ; \eta_{1}\right) \sigma_{0}\left(x_{2} ; \eta_{2}\right)\right\rangle=\left(\frac{\sqrt{4 \eta_{1} \eta_{2}}}{\eta_{+}}\right)^{\Delta_{\mathcal{O}}} G_{0}\left(\frac{t_{12}^{2}}{\eta_{+}}, \frac{\vec{x}_{12}^{2}}{\eta_{+}}\right)$

where we set

$\left\langle\sigma_{0}\left(x_{1} ; \eta_{1}\right) \sigma_{0}\left(x_{2} ; \eta_{2}\right)\right\rangle:=\lim _{\substack{\eta_{t 1} \rightarrow \eta_{1} \\ \eta_{t 2} \rightarrow \eta_{2}}}\left\langle\sigma_{0}\left(x_{1} ; \eta_{t 1}, \eta_{1}\right) \sigma_{0}\left(x_{2} ; \eta_{t 2}, \eta_{2}\right)\right\rangle$.

By using this the induced metric is computed as

$$
\begin{aligned}
g_{\eta \eta}(z) & =\frac{\Delta_{\mathcal{O}}}{4 \eta^{2}}, \quad g_{i j}(z)=\frac{-\delta_{i j}}{\eta} G_{0}^{(0,1)}(\overrightarrow{0}), \\
g_{t t}(z) & =\frac{-1}{\eta} G_{0}^{(1,0)}(\overrightarrow{0}) .
\end{aligned}
$$

From the flow equation we find

$$
\begin{aligned}
-\Delta_{\mathcal{O}} F_{0}(0,0 ; 1,1) & -\frac{1}{\eta} F_{0}^{(0,0,1,0)}(0,0 ; 1,1) \\
& =2(D-1) F_{0}^{(0,1,0,0)}(0,0 ; 1,1), \\
\frac{1}{\eta} F_{0}^{(0,0,1,0)}(0,0 ; 1,1) & =-2 F_{0}^{(1,0)}(0,0 ; 1,1),
\end{aligned}
$$

which leads to

$$
-\Delta_{\mathcal{O}}=2(D-1) G_{0}^{(0,1)}(\overrightarrow{0})-2 G_{0}^{(1,0)}(\overrightarrow{0})
$$

By using (B8) we find $G_{0}^{(0,1)}(\overrightarrow{0})=-G_{0}^{(1,0)}(\overrightarrow{0})$, which gives $G_{0}^{(0,1)}(\overrightarrow{0})=\frac{-\Delta_{\mathcal{O}}}{2 D}$. Using these relations we obtain the induced line element as

$$
\mathrm{d} s^{2}=\Delta_{\mathcal{O}} \frac{-\mathrm{d} t^{2}+\mathrm{d} \vec{x}^{2}+\mathrm{d} \tau^{2}}{\tau^{2}}
$$

where we set $\tau^{2}=2 D \eta$. This is the Lorentzian AdS metric.

\section{APPENDIX C: TRANSFORMATION OF THE FLOWED FIELD}

\section{Useful formulas}

We here collect some useful formulas to calculate transformation properties of the flowed field. 


$$
\begin{aligned}
& e^{\eta \vec{\partial}^{2}} x^{i}=\left(x^{i}+2 \eta \partial^{i}\right) e^{\eta \vec{\partial}^{2}}, \\
& e^{\eta \vec{\partial}^{2}} x^{i} x^{j}=\left(x^{i}+2 \eta \partial^{i}\right)\left(x^{j}+2 \eta \partial^{j}\right) e^{\eta \vec{\partial}^{2}}=\left(x^{i} x^{j}+2 \eta\left(x^{i} \partial^{j}+x^{j} \partial^{i}+\delta_{i j}\right)+4 \eta^{2} \partial^{i} \partial^{j}\right) e^{\eta \vec{\partial}^{2}}, \\
& e^{\eta \vec{\partial}^{2}} \vec{x}^{2}=\left(\vec{x}^{2}+4 \eta \vec{x} \cdot \vec{\partial}+2(d-1) \eta+4 \eta^{2} \vec{\partial}^{2}\right) e^{\eta \vec{\partial}^{2}}, \\
& e^{2 \eta \partial_{+} \partial_{-}} x^{+}=\left(x^{+}+2 \eta \partial_{-}\right) e^{2 \eta \partial_{+} \partial_{-}}, \\
& e^{2 \eta \partial_{+} \partial_{-}}\left(x^{+}\right)^{2}=\left(x^{+}+2 \eta \partial_{-}\right)^{2} e^{2 \eta \partial_{+} \partial_{-}}=\left(\left(x^{+}\right)^{2}+4 \eta x^{+} \partial_{-}+4 \eta^{2} \partial_{-}^{2}\right) e^{2 \eta \partial_{+} \partial_{-}}, \\
& e^{2 \eta \partial_{+} \partial_{-}} x^{-}=\left(x^{-}+2 \eta \partial_{+}\right) e^{2 \eta \partial_{+} \partial_{-}}, \\
& e^{2 \eta \partial_{+} \partial_{-}\left(x^{-}\right)^{2}}=\left(x^{-}+2 \eta \partial_{+}\right)^{2} e^{2 \eta \partial_{+} \partial_{-}}=\left(\left(x^{-}\right)^{2}+4 \eta x^{-} \partial_{+}+4 \eta^{2} \partial_{+}^{2}\right) e^{2 \eta \partial_{+} \partial_{-}}, \\
& e^{2 \eta \partial_{+} \partial_{-}} x^{+} x^{-}=\left(x^{+}+2 \eta \partial_{-}\right)\left(x^{-}+2 \eta \partial_{+}\right) e^{2 \eta \partial_{+} \partial_{-}}=\left(x^{+} x^{-}+2 \eta\left(x^{+} \partial_{+}+x^{-} \partial_{-}+1\right)+4 \eta^{2} \partial_{+} \partial_{-}\right) e^{2 \eta \partial_{+} \partial_{-}}, \\
& e^{\epsilon \partial_{-}^{2}} x^{-}=\left(x^{-}+2 \epsilon \partial_{-}\right) e^{\epsilon \partial_{-}^{2}}, \\
& e^{\epsilon \partial_{-}^{2}}\left(x^{-}\right)^{2}=\left(x^{-}+2 \epsilon \partial_{-}\right)^{2} e^{\epsilon \partial_{-}^{2}}\left(\left(x^{-}\right)^{2}+2 \epsilon\left(2 x^{-} \partial_{-}+1\right)+4 \epsilon^{2} \partial_{-}^{2}\right) e^{\epsilon \partial_{-}^{2}}, \\
& e^{\epsilon \partial_{-}^{2}} x^{+} x^{-}=x^{+}\left(x^{-}+2 \epsilon \partial_{-}\right) e^{\epsilon \partial_{-}^{2}}, \\
& e^{2 \eta \partial_{+} \partial_{-}+\epsilon \partial_{-}^{2}} x^{-}=\left(x^{-}+2 \eta \partial_{+}+2 \epsilon \partial_{-}\right) e^{2 \eta \partial_{+} \partial_{-}+\epsilon \partial_{-}^{2}}, \\
& e^{2 \eta \partial_{+} \partial_{-}+\epsilon \partial_{-}^{2}}\left(x^{-}\right)^{2}=\left(\left(x^{-}\right)^{2}+4 \eta x^{-} \partial_{+}+4 \eta^{2} \partial_{+}^{2}+4 \eta \epsilon \partial_{+} \partial_{-}+2 \epsilon\left(2 x^{-} \partial_{-}+1\right)+4 \epsilon^{2} \partial_{-}^{2}\right) e^{2 \eta \partial_{+} \partial_{-}+\epsilon \partial_{-}^{2}}, \\
& e^{2 \eta \partial_{+} \partial_{-}+\epsilon \partial_{-}^{2}} x^{+} x^{-}=\left(x^{+} x^{-}+2 \eta\left(x^{-} \partial_{-}+x^{+} \partial_{+}+1\right)+4 \eta^{2} \partial_{+} \partial_{-}+4 \eta \epsilon \partial_{-}^{2}+2 \epsilon x^{+} \partial_{-}\right) e^{2 \eta \partial_{+} \partial_{-}+\epsilon \partial_{-}^{2}}, \\
& e^{a \partial_{+}}\left(x^{+}\right)^{n}=\left(x^{+}+a\right)^{n} e^{a \partial_{+}} .
\end{aligned}
$$

\section{General transformation properties}

Under the conformal transformation, the general flowed field $\phi_{\epsilon, m}(x ; \eta)$ transforms as

$$
\begin{gathered}
\delta^{\text {conf }} \phi_{\epsilon, m}(x ; \eta):=e^{\eta\left(\vec{\partial}^{2}+2 \partial_{+} \partial_{-}\right)+\epsilon \partial_{-}^{2}+i \eta 2 \bar{m} \partial_{+}} \delta^{\mathrm{conf}} O(x)=\delta^{\mathrm{conf}} \phi_{\epsilon, m}(x ; \eta)+\Delta^{\mathrm{conf}} \phi_{\epsilon, m}(x ; \eta), \\
\Delta^{\mathrm{conf}} \phi_{\epsilon, m}(x ; \eta):=\left(\delta^{\epsilon}+\delta^{\eta}+\delta^{\eta^{2}}+\delta^{\epsilon \eta}\right) \phi_{\epsilon, m}(x ; \eta),
\end{gathered}
$$

where

$$
\begin{gathered}
\delta^{\mathrm{conf}}:=-\left[a \cdot \partial+\omega^{\mu}{ }_{\nu} \bar{x}^{\nu} \partial_{\mu}+\lambda\left(\bar{x} \cdot \partial+\Delta_{\mathcal{O}}\right)+\bar{x}^{2} b \cdot \partial-2 b \cdot \bar{x}\left(\bar{x} \cdot \partial+\Delta_{\mathcal{O}}\right)\right], \\
\delta^{\eta}:=2 \eta(2 b \cdot \bar{x}-\lambda)\left(\partial_{\eta}-2 i \bar{m} \partial_{+}\right)-2 \eta\left(d-1-2 \Delta_{\mathcal{O}}\right) b \cdot \partial, \\
\delta^{\epsilon}:=2 \epsilon\left[\left(2 b \cdot \bar{x}-\lambda-\omega^{-+}\right) \partial_{-}^{2}+2\left(\bar{x} \cdot \partial+\Delta_{\mathcal{O}}+1\right) b^{+} \partial_{-}-\omega^{i+} \partial_{i} \partial_{-}-2 \bar{x}^{+} \partial_{-} b \cdot \partial\right], \\
\delta^{\eta^{2}}:=4 \eta^{2} b \cdot \partial\left(\partial_{\eta}-2 i \bar{m} \partial_{+}\right), \\
\delta^{\epsilon \eta}:=8 \epsilon \eta b^{+} \partial_{-}\left(\partial_{\eta}-2 i \bar{m} \partial_{+}\right), \\
\delta^{\epsilon^{2}}:=8 \epsilon^{2} b^{+} \partial_{-}^{3},
\end{gathered}
$$

and $\bar{x}=\left(\vec{x}, x^{+}+2 i \bar{m} \eta, x^{-}\right)$with $a \cdot \partial:=a^{i} \partial_{i}+a^{+} \partial_{+}+a^{-} \partial_{-}$. 
[1] J. M. Maldacena, The large N limit of superconformal field theories and supergravity, Int. J. Theor. Phys. 38, 1113 (1999); Adv. Theor. Math. Phys. 2, 231 (1998).

[2] S. S. Gubser, I. R. Klebanov, and A. M. Polyakov, Gauge theory correlators from non-critical string theory, Phys. Lett. B 428, 105 (1998).

[3] E. Witten, Anti-de Sitter space and holography, Adv. Theor. Math. Phys. 2, 253 (1998).

[4] G. 't Hooft, Dimensional reduction in quantum gravity, Conf. Proc. C930308, 284 (1993).

[5] L. Susskind, The World as a hologram, J. Math. Phys. (N.Y.) 36, 6377 (1995).

[6] S. A. Hartnoll, Lectures on holographic methods for condensed matter physics, Classical Quantum Gravity 26, 224002 (2009).

[7] C. P. Herzog, Lectures on holographic superfluidity and superconductivity, J. Phys. A 42, 343001 (2009).

[8] J. McGreevy, Holographic duality with a view toward manybody physics, Adv. High Energy Phys. 2010, 723105 (2010).

[9] A. S. T. Pires, Ads/CFT correspondence in condensed matter, arXiv:1006.5838.

[10] S. Sachdev, Condensed matter and AdS/CFT, Lect. Notes Phys. 828, 273 (2011).

[11] N. Iqbal, H. Liu, and M. Mezei, Lectures on holographic non-Fermi liquids and quantum phase transitions, in String Theory And Its Applications: TASI 2010 From meV to the Planck Scale (2012), pp. 707-815, https://doi.org/10.1142/ 9789814350525_0013.

[12] C. R. Hagen, Scale and conformal transformations in galilean-covariant field theory, Phys. Rev. D 5, 377 (1972); $\mathrm{U}$. Niederer, The maximal kinematical invariance group of the free Schrodinger equation, Helv. Phys. Acta 45, 802 (1972).

[13] M. Henkel, On the two point correlation function in dynamical scaling and Schrodinger invariance, Int. J. Mod. Phys. C 03, 1011 (1992).

[14] M. Henkel, Schrodinger invariance in strongly anisotropic critical systems, J. Stat. Phys. 75, 1023 (1994).

[15] M. Henkel, Local Scale Invariance and Strongly Anisotropic Equilibrium Critical Systems, Phys. Rev. Lett. 78, 1940 (1997).

[16] M. Henkel and J. Unterberger, Schrodinger invariance and space-time symmetries, Nucl. Phys. B660, 407 (2003).

[17] Y. Nishida and D. T. Son, Nonrelativistic conformal field theories, Phys. Rev. D 76, 086004 (2007).

[18] D. T. Son, Toward an AdS/cold atoms correspondence: A geometric realization of the Schrodinger symmetry, Phys. Rev. D 78, 046003 (2008).
[19] K. Balasubramanian and J. McGreevy, Gravity Duals for Non-Relativistic CFTs, Phys. Rev. Lett. 101, 061601 (2008).

[20] S. Kachru, X. Liu, and M. Mulligan, Gravity duals of Lifshitz-like fixed points, Phys. Rev. D 78, 106005 (2008).

[21] S. Aoki and S. Yokoyama, Flow equation, conformal symmetry, and anti-de Sitter geometry, Prog. Theor. Exp. Phys. 2018, $031 \mathrm{~B} 01$ (2018).

[22] S. Aoki and S. Yokoyama, AdS geometry from CFT on a general conformally flat manifold, Nucl. Phys. B933, 262 (2018).

[23] S. Aoki, K. Kikuchi, and T. Onogi, Geometries from field theories, Prog. Theor. Exp. Phys. 2015, 101B01 (2015).

[24] S. Aoki, J. Balog, T. Onogi, and P. Weisz, Flow equation for the large $N$ scalar model and induced geometries, Prog. Theor. Exp. Phys. 2016, 083 B04 (2016).

[25] S. Aoki, J. Balog, T. Onogi, and P. Weisz, Flow equation for the scalar model in the large $N$ expansion and its applications, Prog. Theor. Exp. Phys. 2017, 043B01 (2017).

[26] M. Taylor, Non-relativistic holography, arXiv:0812.0530.

[27] V. Balasubramanian, P. Kraus, and A. E. Lawrence, Bulk versus boundary dynamics in anti-de Sitter space-time, Phys. Rev. D 59, 046003 (1999).

[28] T. Banks, M. R. Douglas, G. T. Horowitz, and E. J. Martinec, AdS dynamics from conformal field theory, arXiv:hep-th/ 9808016.

[29] V. Balasubramanian, P. Kraus, A. E. Lawrence, and S. P. Trivedi, Holographic probes of anti-de Sitter space-times, Phys. Rev. D 59, 104021 (1999).

[30] D. Marolf, States and boundary terms: Subtleties of Lorentzian AdS/CFT, J. High Energy Phys. 05 (2005) 042.

[31] A. Donos and J. P. Gauntlett, Lifshitz solutions of $D=10$ and D=11 supergravity, J. High Energy Phys. 12 (2010) 002.

[32] G. T. Horowitz and B. Way, Lifshitz singularities, Phys. Rev. D 85, 046008 (2012).

[33] M. Taylor, Lifshitz holography, Classical Quantum Gravity 33, 033001 (2016).

[34] A. Hamilton, D. N. Kabat, G. Lifshitz, and D. A. Lowe, Holographic representation of local bulk operators, Phys. Rev. D 74, 066009 (2006).

[35] S. de Haro, S. N. Solodukhin, and K. Skenderis, Holographic reconstruction of space-time and renormalization in the AdS/CFT correspondence, Commun. Math. Phys. 217, 595 (2001).

[36] S. Aoki, J. Balog, and S. Yokoyama, Holographic computation of quantum corrections to the bulk cosmological constant, arXiv:1804.04636. 